\title{
Steady and transient heat transfer analysis using a stable node-based smoothed finite element method
}

\author{
X.Y.Cui ${ }^{1,2^{*}}$, Z.C. Li ${ }^{1,2}$, H. Feng ${ }^{1,2}$, S.Z. Feng ${ }^{3}$
}

\begin{abstract}
In order to cure the instability of NS-FEM and further improve the accuracy, a stable node-based smoothed finite element method (SNS-FEM) is formulated for steady and transient heat transfer problems using linear triangular and tetrahedron element. In present method, both smoothed temperature gradient and variance of temperature gradient in smoothing domains are considered. The accuracy, computational efficiency and stability of SNS-FEM are examined through several numerical examples with different kinds of boundary conditions. It is found that present method is more accurate and efficient than traditional finite element method (FEM) and NS-FEM. Most importantly, compared with NS-FEM, present SNS-FEM can be very stable when dealing with transient heat transfer problems.
\end{abstract}

Key words: Heat transfer; Gradient smoothing; Nodal integration; Stability 


\section{Introduction}

The analysis of heat transfer problems is of great importance in practical engineering areas. As analytical solutions for these problems with complex geometry and boundary conditions are usually not available, numerical methods have been developed and extended for heat transfer problems in the past several decades.

The finite element method (FEM) has been used for heat transfer problems for a long time [1]. Donea and Giuliani [2] have utilized an FEM based iterative method for numerical solution of nonlinear heat transfer problems. Payre et al. [3] has developed an 'upwind' finite element method via numerical integration to improve previous upwind finite element schemes for diffusion-convection problems. The boundary element method (BEM) has been used by Skerget et al. [4] to solve transient heat transfer in reactor solids. The element free Galerkin (EFG) method has been applied to analyze heat transfer problems by Singh et al. [5-7]. Cheng et al. [8] applied the reproducing kernel particle method (RKPM) to two-dimensional unsteady heat conduction problems. MLPG method for two-dimensional steady-state heat conduction problems has been investigated by $\mathrm{Wu}$ et al. [9]. Feng et al. [10] has applied FS-PIM to analyze nonlinear heat conduction in multi-material bodies.

Among all these numerical methods mentioned above, finite element method is the most widely used numerical method with many commercial software packages available. However, the FEM always provide a poor accuracy when low-order linear element is used. Besides, FEM will suffer a significant accuracy loss when the element mesh is heavily distorted due to its strong reliance on element meshes. In the 
FEM framework, researchers have studied and developed many modified numerical algorithms to circumvent some of the defects and great progress has been made. Chen et al. [11] proposed the concept of strain smoothing technique, Liu et al. [12] have introduced this gradient smoothing technique into FEM framework. A class of smoothed FEM models have been developed on the base of the gradient smoothing technique, such as the smoothed finite element method (SFEM) (Liu et al. [13], Xue et al. [14]), the node-based smoothed finite element method (NS-FEM) (Liu et al. [15]), the edge-based smoothed finite element method (ES-FEM) ( Liu et al. [16], Cui et al. [17,18], Feng et al. [19]) and the face-based smoothed finite element method (FS-FEM) (Feng et al. [20]). NS-FEM wins the favor recently for its prominent inherent properties: (1) can give an upper bound solution in energy norm for problems with homogeneous essential boundary conditions; (2) can use polygonal elements with an arbitrary number of sides; (3) well immune from volumetric locking; (4) insensitive to element distortion. Besides, researchers have studied the computation time and computation efficiency of NS-FEM in previous works and have found that the computational efficiency of NS-FEM is about three times lower than that of FEM-T3 in terms of displacement norm and 20 times higher in strain energy norm.

In the other front of development, Liu et al.[21] and Zhang et al. [22] have applied the nodal integration and strain smoothing technique into the point interpolation method (PIM), resulting the linearly conforming point interpolation method (LC-PIM or NS-PIM). The NS-PIM has been applied to steady heat transfer problems by $\mathrm{Wu}$ [23-25]. The NS-PIM using linear shape function is equivalent to the 
NS-FEM based on 3-node triangular element. The NS-PIM or NS-FEM has the same prominent properties in the analysis of steady heat transfer problems as in analysis of solid mechanics problems. However, the excessive node-based smoothing operation results "under-integration" of the weak form and makes the system stiffness "overly-soft", resulting the temporal instability of the NS-FEM when solving transient heat transfer problems.

The temporal instability of nodal integration is firstly found in mesh-free method known as the nodal integration of the element free Galerkin method [26]. Beissel and Belytschko treated it by adding a squared-residual of the equilibrium equation to the potential energy functional as a stabilization term. Zhang et al. [27], Feng et al. [28] and Wang et al. [29] have further applied this technique to the NS-FEM solutions. A parameter $\alpha$ is introduced in this stabilization process to adjust the stiffness of system. And researchers have found it effective using linear elements. To strengthen the "overly-soft" stiffness of NS-FEM, other researchers have formulated the alpha finite element method ( $\alpha$ FEM) (Liu et al. [30]) as well as the hybrid smoothed finite element method (HS-FEM) (Xu et al. [31], Li et al. [32]). They found that these methods can provide "ultra-accurate" numerical solutions with a proper parameter $\alpha$. The parameter $\alpha$ plays an important role in all the methods mentioned above. Its value can greatly influence the numerical results. However, the value of $\alpha$ is affected by different factors, such as the size of mesh discretization and the nature of the problem. Until now, researchers have not found an optimal $\alpha$ value suitable for all problems.

In this work, a new stable node-based smoothed finite element method 
(SNS-FEM) is formulated for steady and transient heat transfer problems using linear triangular and tetrahedron element. The temperature gradient smoothing operation is firstly implemented within each smoothing domain as in NS-FEM, and then the temperature gradient is expanded at first order by Taylor equation. In each smoothing domain, both smoothed temperature gradient and variance of temperature gradient are considered. Numerical examples with different kinds of boundary conditions are presented to examine the stability, accuracy, as well as efficiency of present method through comparing the results with those obtained by the FEM and the original NS-FEM. Results show that present SNS-FEM can provide stable solutions for transient heat transfer problems, and performs better in accuracy and efficiency than traditional FEM and original NS-FEM.

\section{Discretized system equations}

\subsection{Standard Galerkin weak form}

To note that, 3D problem will be discussed in detail in the following, and in 2D equations, terms with respect to $z$ or $w$ will vanish, which can be easily obtained following the procedure.

Consider a three-dimensional heat transfer domain $\Omega$ bounded by $\Gamma$ in which the governing equation is written as:

$$
\begin{aligned}
\frac{\partial}{\partial x}\left(k_{x} \frac{\partial T}{\partial x}\right)+\frac{\partial}{\partial y}\left(k_{y} \frac{\partial T}{\partial y}\right)+\frac{\partial}{\partial z}\left(k_{z} \frac{\partial T}{\partial z}\right) & \\
+Q(x, y, z, t) & =\rho c \frac{\partial T(x, y, z, t)}{\partial t}
\end{aligned}
$$

The initial condition 


$$
T=T_{0}
$$

Initial condition

The boundary conditions

$$
\begin{array}{cc}
T=T_{\Gamma} & \text { Dirichlet boundary } \\
-k_{x} \frac{\partial T}{\partial x} n_{x}-k_{y} \frac{\partial T}{\partial y} n_{y}-k_{z} \frac{\partial T}{\partial z} n_{z}=q & \text { Neumann boundary } \\
-k_{x} \frac{\partial T}{\partial x} n_{x}-k_{y} \frac{\partial T}{\partial y} n_{y}-k_{z} \frac{\partial T}{\partial z} n_{z}=h\left(T-T_{a}\right) & \text { Robin boundary } \\
k_{x} \frac{\partial T}{\partial x} n_{x}+k_{y} \frac{\partial T}{\partial y} n_{y}+k_{z} \frac{\partial T}{\partial z} n_{z}=\mathbf{0} & \text { Adiabatic boundary }
\end{array}
$$

Where $k_{x}, k_{y}$ and $k_{z}$ are the thermal conductivities, $Q(x, y, z, t)$ is the internal heat source, $T(x, y, z, t)$ is the temperature of point $(x, y, z)$ at time $t, \rho$ is the density, $c$ is the specific heat, $n$ is the unit normal vector to the boundary, $q$ is the prescribed heat flux, $h$ is the convective heat transfer coefficient and $T_{a}$ is the temperature of surrounding medium.

The standard Galerkin weak form can be writted as:

$$
\begin{aligned}
& \int_{\Omega} \rho c \delta T^{T} \frac{\partial T}{\partial t} d \Omega+\int_{\Omega}(\mathbf{L} \delta T)^{T}\left[\begin{array}{ccc}
k_{x} & 0 & 0 \\
0 & k_{y} & 0 \\
0 & 0 & k_{z}
\end{array}\right](\mathbf{L} T) d \Omega-\int_{\Omega} \delta T^{T} Q d \Omega \\
& +\int_{\Gamma_{q}} \delta T^{T} q d \Gamma+\int_{\Gamma_{h}} \delta T^{T} h\left(T-T_{a}\right) d \Gamma=0
\end{aligned}
$$

Where $\mathbf{L}$ is a differential operator in the following form

$$
\mathbf{L}=\left[\begin{array}{c}
\frac{\partial}{\partial x} \\
\frac{\partial}{\partial y} \\
\frac{\partial}{\partial z}
\end{array}\right]
$$

In the above Galerkin weak form Eq. (7), the temperature field $T$ can be expressed in an approximate form: 


$$
T=\sum_{i=1}^{m} N_{i} T_{i}
$$

Where $T_{i}$ is the unknown temperature at the node $i$, and $N_{i}$ is the shape function.

Substituting Eq. (9) into Eq. (7), Then Eq. (7) can be expressed as:

$$
\begin{aligned}
& \int_{\Omega} \rho c \mathbf{N}^{T} \mathbf{N} \frac{\partial \mathbf{T}}{\partial t} d \mathbf{\Omega}+\int_{\Omega}\left[\left(\frac{\partial \mathbf{N}}{\partial x}\right)^{T} k_{x} \frac{\partial \mathbf{N}}{\partial x}+\left(\frac{\partial \mathbf{N}}{\partial y}\right)^{T} k_{y} \frac{\partial \mathbf{N}}{\partial y}+\left(\frac{\partial \mathbf{N}}{\partial z}\right)^{T} k_{z} \frac{\partial \mathbf{N}}{\partial z}\right] \mathbf{T} d \mathbf{\Omega} \\
& +\int_{\Gamma_{2}} \mathbf{N}^{T} q d \Gamma+\int_{\Gamma_{3}} \mathbf{N}^{T} \mathbf{N} \mathbf{T} d \Gamma-h \int_{\Gamma_{3}} \mathbf{N}^{T} T_{a} d \Gamma-\int_{\Omega} \mathbf{N}^{T} Q d \Omega=0
\end{aligned}
$$

The discretized system equilibrium equation can be finally expressed in the following matrix form:

$$
\mathbf{M}\left\{\frac{\partial \mathbf{T}}{\partial t}\right\}+\left[\mathbf{K}+\mathbf{K}^{h}\right]\{\mathbf{T}\}=\{\mathbf{P}\}
$$

In which

$$
\begin{gathered}
\mathbf{M}_{i j}=\rho c \int_{\Omega} \mathbf{N}_{i}^{T} \mathbf{N}_{j} d \Omega \\
\mathbf{K}_{i j}=\int_{\Omega} \mathbf{B}_{i}^{T} \mathbf{D} \mathbf{B}_{j} d \Omega \\
\mathbf{B}_{i}=\mathbf{L} N_{i}=\left[\begin{array}{c}
N_{i, x} \\
N_{i, y} \\
N_{i, z}
\end{array}\right] \\
\mathbf{D}=\left[\begin{array}{ccc}
k_{x} & 0 & 0 \\
0 & k_{y} & 0 \\
0 & 0 & k_{z}
\end{array}\right] \\
\mathbf{K}_{i j}^{h}=\int_{\Gamma_{3}} h \mathbf{N}_{i}^{T} \mathbf{N}_{j} d \Gamma_{3} \\
\mathbf{P}_{i}=\int_{\Omega} \mathbf{N}_{i}^{T} Q d \Omega-\int_{\Gamma_{2}} \mathbf{N}_{i}^{T} q d \Gamma+h \int_{\Gamma_{3}} \mathbf{N}_{i}^{T} T_{a} d \Gamma
\end{gathered}
$$

For time approximation, the backward difference technique is used here because of its unconditional stability even using a larger time step. So, $\partial \mathrm{T}(\mathrm{t}) / \partial \mathrm{t}$ can be expressed as: 


$$
\left\{\frac{\partial \mathbf{T}(t)}{\partial t}\right\}=\frac{\{\mathbf{T}(t)\}-\{\mathbf{T}(t-\Delta t)\}}{\Delta t}+o(\Delta t)
$$

Substituting Eq. (18) into Eq. (11), we obtain the following equation

$$
\left(\mathbf{K}+\mathbf{K}^{h}+\frac{\mathbf{M}}{\Delta t}\right)\{\mathbf{T}(t)\}=\mathbf{P}+\left(\frac{\mathbf{M}}{\Delta t}\right)\{\mathbf{T}(t-\Delta t)\}
$$

\subsection{Nodal integration with a stabilization term}

\subsubsection{Nodal integration using gradient smoothing technique}

To carry out the NS-FEM, a background mesh of tetrahedrons (or triangles for 2D) with a total number of $N$ nodes is firstly generated. Based on the background mesh, the problem domain $\Omega$ is then partitioned into $N$ non-overlapping smoothing domains of polyhedron (or polygon for 2D) $\Omega_{k}(k=1,2, \ldots, N)$ centered by node $k$.

Fig.1 shows a typical smoothing domain in $2 \mathrm{D}$ and $3 \mathrm{D}$ domain. $\Gamma_{k}$ is the boundary of the smoothing domain $\Omega_{k}$, and the union of all smoothing domains forms exactly the global domain $\Omega$.

Using gradient smoothing technique, the temperature gradient in domain $\Omega_{k}$ can be written as:

$$
\begin{gathered}
\Delta \bar{T}=\overline{\mathbf{g}}\left(X_{k}\right)=\frac{1}{A_{k}} \int_{\Omega_{k}} \mathbf{g}\left(X_{k}\right) d \Omega=\sum_{i \in D_{k}} \overline{\mathbf{B}}_{i} \mathbf{T}_{i} \\
\overline{\mathbf{B}}_{i}^{T}\left(X_{k}\right)=\left[\begin{array}{lll}
\bar{b}_{i x} & \bar{b}_{i y} & \bar{b}_{i z}
\end{array}\right] \\
\bar{b}_{i p}=\frac{1}{V_{k}} \int_{\Gamma_{k}} N_{i}(X) n_{p}(X) d \Gamma \quad(p=x, y, z)
\end{gathered}
$$

Where $\mathrm{V}_{k}$ is the volume of the smoothing domain $\Omega_{k}, \mathrm{D}_{k}$ is the total number of nodes in the support domain of node $k$ and $N_{i}(X)$ is the shape function for node $i$.

As a linear compatible temperature field along the boundary $\Gamma_{k}$ is used, one Gaussian point is sufficient for line integration along the boundary $\Gamma_{k}$ of each 
smoothing domain $\Omega_{k}$.

Then, Eq. (22) can be simplified to its algebraic form:

$$
\bar{b}_{i p}\left(X_{k}\right)=\frac{1}{V_{k}} \sum_{j=1}^{N_{s}} n_{j p} N_{i}\left(X_{j}^{G P}\right) A_{j} \quad(p=x, y, z)
$$

$N_{s}$ is the total number of segments of the boundary $\Gamma_{k}, \mathrm{X}_{j}^{\mathrm{GP}}$ is the central point (Gaussian point) of the boundary segment $\Gamma_{j}$, the outward unit normal and area of which are denoted as $n_{j p}$ and $\mathrm{A}_{j}$, respectively.

So, the smoothed stiffness matrix of the smoothing domain $\Omega_{k}$ can be expressed as

$$
\overline{\mathbf{K}}_{i j}^{k}=\left(\overline{\mathbf{B}}_{i}^{k}\right)^{T}\left[\begin{array}{ccc}
k_{x} & 0 & 0 \\
0 & k_{y} & 0 \\
0 & 0 & k_{z}
\end{array}\right] \overline{\mathbf{B}}_{j}^{k} V_{k}
$$

\subsubsection{A stabilization term}

In the original NS-FEM, the smoothed temperature gradient of each node $k$ is selected to represent the temperature gradient of each smoothing domain to perform the integration, as shown in Fig. 2a and Fig. 2c. The overly node-based gradient smoothing operation of the NS-FEM results under-integration of the weak form and makes the system stiffness "overly-soft", leading to temporal instability in analysis of transient heat transfer problems. In order to strengthen the "overly-soft" NS-FEM model, the variance of temperature gradient in each node-based smoothing domain is also taken into consideration in present SNS-FEM. Firstly, we approximate the smoothing domain $\Omega_{k}$ as a sphere (or circular for 2D) domain $\Omega_{k}^{c}$ with the same volume (or area ) $\mathrm{V}_{k}$. Then, we subdivide the approximate domain into six (or four) equal sub-domains. Six (or four) intersections of the boundary of the sphere (or 
circular) domain and coordinate axes are selected as integration points, just as shown in Fig. $2 \mathrm{~b}$ and Fig.2d.

The radius of the approximate domain $\Omega_{k}^{c}$ is expressed as

$$
\begin{array}{ll}
l_{c}=\sqrt{A_{k} / \pi} & \text { for } 2 \mathrm{D} \\
l_{c}=\sqrt[3]{3 V_{k} / 4 \pi} & \text { for } 3 \mathrm{D}
\end{array}
$$

Assuming temperature gradient in $\Omega_{k}^{c}$ is continuous and derivable at the first order, we expand it by Taylor formula as:

$$
\mathbf{g}^{c} \approx \overline{\mathbf{g}}_{k}+\frac{\partial \mathbf{g}^{c}}{\partial x}\left(x-x_{k}\right)+\frac{\partial \mathbf{g}^{c}}{\partial y}\left(y-y_{k}\right)+\frac{\partial \mathbf{g}^{c}}{\partial z}\left(y-y_{z}\right)
$$

So temperature gradient $\mathbf{g}$ at the six integration points are expressed as

$$
\begin{aligned}
& \mathbf{g}_{1}^{c}=\overline{\mathbf{g}}_{k}-\frac{\partial \mathbf{g}^{c}}{\partial x} l_{c}, \mathbf{g}_{2}^{c}=\overline{\mathbf{g}}_{k}-\frac{\partial \mathbf{g}^{c}}{\partial y} l_{c}, \mathbf{g}_{3}^{c}=\overline{\mathbf{g}}_{k}-\frac{\partial \mathbf{g}^{c}}{\partial z} l_{c} \\
& \mathbf{g}_{4}^{c}=\overline{\mathbf{g}}_{k}+\frac{\partial \mathbf{g}^{c}}{\partial x} l_{c}, \mathbf{g}_{5}^{c}=\overline{\mathbf{g}}_{k}+\frac{\partial \mathbf{g}^{c}}{\partial y} l_{c}, \mathbf{g}_{6}^{c}=\overline{\mathbf{g}}_{k}+\frac{\partial \mathbf{g}^{c}}{\partial z} l_{c}
\end{aligned}
$$

Substituting Eq. (27) into the Galerkin weak form, the smoothed stiffness matrix over the smoothing domain $\Omega_{k}$ can be then modified to the following form

$$
\mathbf{K}_{k}^{c}=\overline{\mathbf{B}}_{k}^{T} \mathbf{D} \overline{\mathbf{B}}_{k} V_{k}+\left(\overline{\mathbf{B}}_{k}^{T}\right)_{x} \mathbf{D}\left(\overline{\mathbf{B}}_{k}\right)_{x} \frac{V_{k}}{3}+\left(\overline{\mathbf{B}}_{k}^{T}\right)_{y} \mathbf{D}\left(\overline{\mathbf{B}}_{k}\right)_{y} \frac{V_{k}}{3}+\left(\overline{\mathbf{B}}_{k}^{T}\right)_{z} \mathbf{D}\left(\overline{\mathbf{B}}_{k}\right)_{z} \frac{V_{k}}{3}
$$

The last three items at the right hand of Eq. (28) is the stabilization term of the original NS-FEM for 3D problems.

For 2D problems

$$
\mathbf{K}_{k}^{c}=\overline{\mathbf{B}}_{k}^{T} \mathbf{D} \overline{\mathbf{B}}_{k} A_{k}+\left(\overline{\mathbf{B}}_{k}^{T}\right)_{x} \mathbf{D}\left(\overline{\mathbf{B}}_{k}\right)_{x} \frac{A_{k}}{2}+\left(\overline{\mathbf{B}}_{k}^{T}\right)_{y} \mathbf{D}\left(\overline{\mathbf{B}}_{k}\right)_{y} \frac{A_{k}}{2}
$$

And the last two items at the right hand of Eq. (29) is the stabilization term of the original NS-FEM for 2D problems.

\section{Numerical examples}

Four numerical examples here are investigated to examine the property of the 
SNS-FEM in analysis of steady and transient heat transfer problems. For comparison, the traditional FEM and original NS-FEM are also adopted to evaluate the same problem using the same tetrahedral (or triangular) mesh as present method. To study the convergence, the thermal equivalent energy [33] is defined as follows

$$
U_{T}=\int_{\Omega} \mathbf{g}^{T}\left[\begin{array}{ccc}
k_{x} & 0 & 0 \\
0 & k_{y} & 0 \\
0 & 0 & k_{y}
\end{array}\right] \mathbf{g} d \Omega
$$

To study the efficiency of present method, an error norm in temperature is defined as

$$
E_{t}=\sqrt{\frac{\sum_{i=1}^{N}\left(T_{i}^{\text {exact }}-T_{i}^{\text {num }}\right)^{2}}{\sum_{i=1}^{N}\left(T_{i}^{\text {exact }}\right)^{2}}}
$$

Where 'exact' denotes the exact value of the problem, and 'num' represents a numerical solution obtained by a numerical method including the FEM, NS-FEM and present SNS-FEM.

When the analytical solutions are not available, we use the finite element commercial software ABAQUS with very fine meshes to obtain the reference solutions for these complex problems.

\subsection{Example 1}

First, present SNS-FEM is applied to a steady heat transfer problem to examine its accuracy and convergence. The geometry and boundary conditions of the problem are shown in Fig. 3. The related parameters used in the computation are taken as $l_{1}=0.32 \mathrm{~m}, \quad l_{2}=0.08 \mathrm{~m}, \quad \mathrm{OA}=0.16 \mathrm{~m}, \mathrm{AB}=0.06 \mathrm{~m}, \mathrm{CD}=0.08 \mathrm{~m}, \quad k_{x}=k_{y}=10 \mathrm{~W} /\left(\mathrm{m} \cdot{ }^{\circ} \mathrm{C}\right)$, $q=-5000 \mathrm{~W} / \mathrm{m}^{2}, T_{\Gamma}=100$. As analytical solution is not available here, we use the 
software ABAQUS with very fine mesh of 41345 nodes to obtain the reference solution for comparison.

Fig. 4 and Fig. 5 show the convergence of temperature versus the number of degrees of freedom at reference points $\mathrm{A}$ and $\mathrm{B}$. It can be clearly observed that temperatures computed by present method are closer to reference solutions than that computed by FEM and the original NS-FEM with the same number of degree of freedom. Fig. 6 shows the comparison of temperature gradient in x-direction obtained by present SNS-FEM, FEM, and NS-FEM using the same triangular mesh of 1111 nodes. As we know, the FEM using linear triangular elements will produce constant temperature gradient field within the elements, possibly leading to inaccurate results, especially in high temperature gradient domains of the problem. The node-based smoothing operation makes the NS-FEM and present SNS-FEM produce better gradient field in high gradient regions, as shown in Fig. 6. However, the temperature gradient contour produced by NS-FEM is not smooth enough. It can be seen that results produced by the SNS-FEM is smoother than that of NS-FEM and can better agree with the reference solution.

Fig. 7 shows the convergence of equivalent energy of the system versus the number of degrees of freedom. As is known, due to the "overly-stiff" property of the FEM and the "overly-soft" property of the NS-FEM, they separately obtain a lower and an upper bound solution in energy norm to elasticity problems. These properties of lower and upper bound solutions in thermal equivalent energy norm have also been studied in steady heat transfer problems by Wu et al. [18-20]. The SNS-FEM, as we 
have said, can strengthen the system stiffness of the original NS-FEM, and is more likely to have a "close-to-exact" stiffness of the system. So, thermal equivalent energy of the system computed by present SNS-FEM should be more close to the reference solution, just as shown in Fig. 7. The results show that thermal equivalent energy of present SNS-FEM converges faster than that of standard FEM and original NS-FEM.

\subsection{Example 2}

To further study the applicability of present SNS-FEM in transient heat transfer problems, a square plate as shown in Fig. 8 is then considered. The related parameters used in the computation are taken as $l=\pi, k_{x}=1 \mathrm{~W} /\left(\mathrm{m} \cdot{ }^{\circ} \mathrm{C}\right), k_{y}=1 \mathrm{~W} /\left(\mathrm{m} \cdot{ }^{\circ} \mathrm{C}\right), \rho=1 \mathrm{~kg} / \mathrm{m}^{3}$, $c=1 \mathrm{~J} /(\mathrm{kg} \cdot), Q=0 \mathrm{~W} / \mathrm{m}^{3}$. The boundary conditions of this transient heat transfer problem are written as $\mathrm{T}(x, 0, t)=\mathrm{T}(0, y, t)=\mathrm{T}(l, y, t)=\mathrm{T}(x, l, t)=0^{\circ} \mathrm{C}$, and the initial condition for this problem is $\mathrm{T}(x, y, 0)=10 \sin (x) \cdot \sin (y)$. The analytical solution is $\mathrm{T}(x, y, t)=$ $10 \sin (x) \cdot \sin (y) \cdot \exp (-2 t)$

To examine the accuracy of present method, the temperature distribution along $y=\pi / 2$ at $\mathrm{t}=3 \mathrm{~s}$ is selected for observation, as shown in Fig. 9. It can be obviously seen from Fig. 9 that results calculated by present method can more accurately match the analytical solution than the FEM and NS-FEM with the same mesh of 181 nodes. It can also be seen in Fig. 9 that temperature along the neutral line computed by NS-FEM does not vary evenly, which indicates the instability of the NS-FEM when dealing with transient heat transfer problems.

Then the comparison of temperature distribution of the whole square plate at $\mathrm{t}=5 \mathrm{~s}$ is plotted, as shown in Fig. 10, from which the instability of the NS-FEM in 
analysis of transient heat transfer problem can be seen obviously. It can also be seen from Fig. 10 that the SNS-FEM can well cure the defect of the temporal instability of the original NS-FEM. Figs. 11-14 plot the temperature history of four reference points using FEM and present SNS-FEM with the same mesh. It can be seen that present SNS-FEM can well deal with transient heat transfer problems and can accurately match the analytical solutions even with a sparse grid.

To examine the convergence of the SNS-FEM, four models with 133, 181, 305, and 461 nodes are created. Fig. 15 shows the comparison of convergence of equivalent energy of the system versus the number of degrees of freedom at $t=2 \mathrm{~s}$. The results also show that the convergence of SNS-FEM is faster than that of FEM and NS-FEM.

Fig. 16 shows the comparison of computational efficiency between FEM, NS-FEM and SNS-FEM. It can be observed that the error norm of present SNS-FEM is smaller than that of FEM and NS-FEM when using the same CPU time. It can also be seen that SNS-FEM costs less CPU time than FEM and NS-FEM for the same value of error norm. From this perspective, the SNS-FEM is more efficient than traditional FEM and NS-FEM.

\subsection{Example 3}

An axisymmetric model, the geometry and boundary conditions of which are shown as Fig. 17, is further studied to validate the applicability of present SNS-FEM in transient heat transfer problems. The related parameters used in this problem are taken as $\mathrm{OA}=0.1 \mathrm{~m}, \mathrm{AB}=0.2 \mathrm{~m}, \mathrm{AL}=0.5 \mathrm{~m}$, and the other lengths of boundaries are 
$0.1 \mathrm{~m} . k_{x}=10 \mathrm{~W} /\left(\mathrm{m} \cdot{ }^{\circ} \mathrm{C}\right), k_{y}=10 \mathrm{~W} /\left(\mathrm{m} \cdot{ }^{\circ} \mathrm{C}\right), q=2000 \mathrm{~W} / \mathrm{m}^{2}, h=1000 \mathrm{~W} /\left(\mathrm{m}^{2} \cdot\right), T_{a}=100$, $\rho=10 \mathrm{~kg} / \mathrm{m}^{3}, c=10 \mathrm{~J} /(\mathrm{kg} \cdot)$, and the initial condition is $T_{t=0}=100$. This problem is analyzed using the FEM, NS-FEM and present SNS-FEM with the same triangular mesh. The reference solution here is obtained using ABAQUS with a very fine mesh of 82080 nodes.

Fig. 18 shows the comparison of temperature distribution of the system at $\mathrm{t}=5 \mathrm{~s}$. It obviously shows that result calculated by the original NS-FEM is not stable, and the SNS-FEM can well eliminate the temporal instability. Then four reference points are selected to further examine the performance of the SNS-FEM in analysis of transient heat transfer problem of this axisymmetric model. Figs. 19-22 plot the temperature history of these reference points using the FEM and the SNS-FEM with the same mesh. These figures also show that present SNS-FEM can well deal with transient heat transfer problems and can provide a "close-to-exact" result even with a sparse grid.

To examine the convergence of the SNS-FEM, four models with 357, 637,991 and 1351 nodes are also created. Fig. 23 shows the convergence of the thermal equivalent energy versus the number of degrees of freedom after the system stabilize. It can be clearly seen that even with a sparse grid, the equivalent energy obtained by SNS-FEM is almost equal to the reference solution. The convergence of SNS-FEM is much faster than that of FEM and NS-FEM.

\subsection{Example 4}

In this section, a 3D piston model is further studied to examine the properties of 
present SNS-FEM in analysis of 3D heat transfer problems. Due to the symmetry condition, only a quarter of the piston model is analyzed. The geometry and mixed thermal boundary conditions of this model are shown in Fig. 24. In the computation, the related parameters are taken as $k_{x}=k_{y}=k_{z}=5 \mathrm{~W} /\left(\mathrm{m} \cdot{ }^{\circ} \mathrm{C}\right), q=-2000 \mathrm{~W} / \mathrm{m}^{2}$, $h=1000 \mathrm{~W} /\left(\mathrm{m}^{2} \cdot \quad\right), T_{a}=400$. This problem is analyzed using the FEM, NS-FEM and present SNS-FEM with the same tetrahedron mesh, and the reference solution here is also obtained using ABAQUS with a very dense hexahedron mesh.

Fig. 25 shows the comparison of temperature distribution along edge CD obtained by FEM, NS-FEM and SNS-FEM with the same mesh of 3497 nodes. It can be observed that result obtained by present SNS-FEM matches the reference solution much better than that of FEM and NS-FEM. Fig. 26 compares the temperature distribution of the quarter piston model. It can also be seen that SNS-FEM can result a better temperature field especially in the bottom zone of the piston. The convergence of temperatures versus the number of degrees of freedom at reference points $\mathrm{B}$ and $\mathrm{F}$ are shown in Fig. 27 and Fig. 28, from which better results of present SNS-FEM can also be obviously seen.

As analytical solution is not available here, it is impossible to use Eq. (31) to study the efficiency of present SNS-FEM. Then, a much denser mesh of 23088 nodes is constructed. Fig. 29 shows the temperature distributions along edge CD obtained by SNS-FEM using 3497 nodes and FEM using 23088 nodes. The CPU time of FEM using 23088 nodes is 76 seconds, and the CPU time of SNS-FEM using 3947 nodes is just 13 seconds. Fig. 29 shows that, even using a much denser mesh, result computed 
by FEM is also less accurate than that of SNS-FEM. So, it can be inferred that, when using FEM, a denser mesh with more than 23088 nodes should be constructed to guarantee the same computational accuracy as SNS-FEM using only 3947 nodes. Then, the CPU time of FEM should be longer than 76 seconds. That is to say, to guarantee the same computational accuracy, the CPU time of SNS-FEM is much less than that of FEM. From this perspective, the SNS-FEM is also more efficient than FEM in analysis of 3D heat transfer problems.

\section{Conclusions}

In this work, a stable NS-FEM is presented for steady and transient heat transfer problems using linear triangular or tetrahedral elements. Some numerical examples with different kinds of boundary conditions are studied to examine the accuracy, efficiency, and stability of the SNS-FEM dealing with steady and transient heat transfer problems. From these studies, several conclusions can be drawn as follows:

(1) The results obtained by present SNS-FEM are more accurate than that of the FEM and original NS-FEM with the same mesh.

(2) The SNS-FEM converges faster than original NS-FEM and the traditional FEM using the same linear elements.

(3) Computational efficiency of present SNS-FEM is higher than that of traditional FEM and original NS-FEM.

(4) In the original NS-FEM, the excessive node-based smoothing operation results "under-integration" of the weak form and makes the system stiffness “overly-soft", resulting the temporal instability of the NS-FEM when solving transient 
heat transfer problems. In present SNS-FEM, the variance of temperature gradient in the smoothing domain is also taken into consideration, so it can strengthen the "overly-soft" stiffness of the original NS-FEM. Numerical examples show that present SNS-FEM can successfully cure the temporal instability of NS-FEM for transient heat transfer problems.

\section{Acknowledgments}

The support of The National Science Foundation of China (11472101) and Postdoctoral Science Foundation of China (2013M531780), State Key Program of National Natural Science of China (61232014) are gratefully acknowledged.

\section{References}

[1] K.J. Bathe, H. Saunders, Finite element procedures in engineering analysis, J. Press. Vessel Technol. 106 (1984) 421.

[2] J. Donea, S.Giuliani, Finite element analysis of steady-state nonlinear heat transfer problems, Nucl. Eng. Des. 30 (1974) 205-213.

[3] Payre G, De Broissia M, Bazinet J, An 'upwind'finite element method via numerical integration, Int. J. Numer. Meth. Eng. 18 (3) (1982) 381-396.

[4] P. Skerget, A. Alujevic, Boundary element method for nonlinear transient heat transfer of reactor solids with convection and radiation on surfaces, Nucl. Eng. Des Nucl. Eng. Des. 76 (1983) 47-54.

[5] I.V. Singh, K. Sandeep, R. Prakash, The element free Galerkin method in three-dimensional steady state heat conduction, Int. J. Comput. Eng. Sci. 3 (2002) 291-303. 
[6] I.V. Singh, M. Tanaka, Heat transfer analysis of composite slabs using meshless element free Galerkin method, Comput. Mech. 38 (2006) 521-532.

[7] A. Singh, I.V. Singh, R. Prakash, Meshless element free Galerkin method for unsteady nonlinear heat transfer problems, Int. J. Heat Mass Transf. 50 (2007) 1212-1219.

[8] R. Cheng, K. Liew, The reproducing kernel particle method for two-dimensional unsteady heat conduction problems, Comput. Mech. 45 (2009) 1-10.

[9] X.H. Wu, S.P. Shen, W.Q. Tao, Meshless local Petrov-Galerkin collocation method for two-dimensional heat conduction problems, CMES, 22 (2007) 65-76.

[10] S.Z. Feng, X.Y. Cui, A.M. Li, G.Z. Xie, A face-based smoothed point interpolation method (FS-PIM) for analysis of nonlinear heat conduction in multi-material bodies, Int. J. Therm. Sci. 100 (2016) 430-437.

[11] J.S. Chen, C.T. Wu, S. Yoon, Y.A. You, A stabilized conforming nodal integration for Galerkin meshfree methods, Int. J. Numer. Meth. Eng. 50 (2) (2001) 435-466.

[12] G. R. Liu, A generalized gradient smoothing technique and the smoothed bilinear form for Galerkin formulation of a wide class of computational methods, Int. J. Comput. Methods, 5 (2008) 199-236.

[13] G.R. Liu, K.Y. Dai, T. Nguyen-Thoi, A smoothed finite element method for mechanics problems, Comput. Mech, 39 (2007) 859-877.

[14] B.Y. Xue, S.C. Wu, W. H. Zhang, G.R. Liu, A smoothed FEM (S-FEM) for heat transfer problems, Int. J. Comput. Methods, 10 (2013) 01-14. 
[15] G. R. Liu, T. Nguyen-Thoi, H. Nguyen-Xuan, K.Y. Lam, A node-based smoothed finite element method (NS-FEM) for upper bound solutions to solid mechanics problems, Comput. Struct. 87 (2009) 14-26.

[16] G.R. Liu, T. Nguyen-Thoi, K.Y. Lam, An edge-based smoothed finite element method (ES-FEM) for static, free and forced vibration analyses of solids, J. Sound Vib. 320 (2009) 1100-1130.

[17] X.Y. Cui, G.R. Liu, G.Y. Li, G.Y. Zhang, G. Zheng, Analysis of plates and shells using an edge-based smoothed finite element method, Comput. Mech, 45 (2010) 141-156.

[18] X.Y. Cui , G.R. Liu, G.Y. Li, G.Y. Zhang, G.Y. Sun, Analysis of elastic-plastic problems using edge-based smoothed finite element method, Int. J. Press Vessel. Pip. 86 (2009) 711-718.

[19] S.Z. Feng, X.Y. Cui, G.Y. Li, Analysis of transient thermo-elastic problems using edge-based smoothed finite element method, Int. J. Therm. Sci. 65 (2013) 127-135.

[20] S.Z. Feng, X.Y. Cui, G.Y. Li, Transient thermal mechanical analyses using a face-based smoothed finite element method (FS-FEM), Int. J. Therm. Sci. 74 (2013) 95-103.

[21] G.R. Liu, Y. Li, K.Y. Dai, Y.Y. Wang, Z.H. Zhong, A linearly conforming point interpolation method (LC-PIM) for 2D solid mechanics problems, Int. J. Comput. Methods, 2 (2006) 645-665.

[22] G.Y. Zhang, G.R. Liu, Y.Y. Wang, H.T. Huang, Z.H. Zhong, G.Y. Li, X. Han, A 
linearly conforming point interpolation method (LC-PIM) for three-dimensional elasticity problems, Int. J. Numer. Methods Eng. 72 (2007) 1524-1543.

[23] S.C. Wu, G.R. Liu, H.O. Zhang, G.Y. Zhang, A node-based smoothed point interpolation method (NS-PIM) for thermoelastic problems with solution bounds, Int. J. Heat Mass Transf. 52 (2009) 1464-1471.

[24] S.C. Wu, G.R. Liu, H.O. Zhang, G.Y. Zhang, A node-based smoothed point interpolation method (NS-PIM) for three-dimensional thermoelastic problems, Numer. Heat Transfer A Appl. 54 (2008) 1121-1147.

[25] S.C. Wu, G.R. Liu, H.O. Zhang, X. Xu, Z.R. Li, A node-based smoothed point interpolation method (NS-PIM) for three-dimensional heat transfer problems, Int. J. Therm. Sci. 48 (2009) 1367-1376.

[26] S. Beissel, T. Belytschko, Nodal integration of the elementfree Galerkin method, Comput. Methods Appl. Mech. Eng. 139 (1996) 49-74.

[27] Z.Q. Zhang, G.R. Liu, Temporal stabilization of the node-based smoothed finite element method and solution bound of linear elastostatics and vibration problems, Comput. Mech, 46 (2010) 229-246.

[28] H. Feng, X.Y. Cui, G.Y. Li, A temporal stable node-based smoothed finite element method for three-dimensional elasticity problems, Comput. Mech, 53 (2014) 859-876.

[29] G. Wang, X.Y. Cui, G.Y. Li, Temporal stabilization nodal integration method for static and dynamic analyses of Reissner-Mindlin plates, Comput. Struct. 152 (2015) $125-141$ 
[30] G.R. Liu, T. Nguyen-Thoi, K.Y. Lam, A novel alpha finite element method $(\alpha \mathrm{FEM})$ for exact solution to mechanics problems using triangular and tetrahedral elements, Comput. Methods Appl. Mech. Eng. 197 (2008) 3883-3897.

[31] X. Xu, Y.T. Gu, G.R. Liu, A hybrid smoothed finite element method (H-SFEM) to solid mechanics problems, Int. J. Comput. Methods, 10 (2013) 1-17.

[32] E. Li, Z.P. Zhang, Z.C. He, X. Xu, G.R. Liu, Q. Li, A Smoothed finite element method with exact solutions in heat transfer problems, Int. J. Heat Mass Transf. 78 (2014) 1219-1231.

[33] O.C. Zienkiewicz, R.L. Taylor, The Finite Element Method (V1: The Basis), 5th ed., Butterworth-Heinemann, Oxford, 2000. 


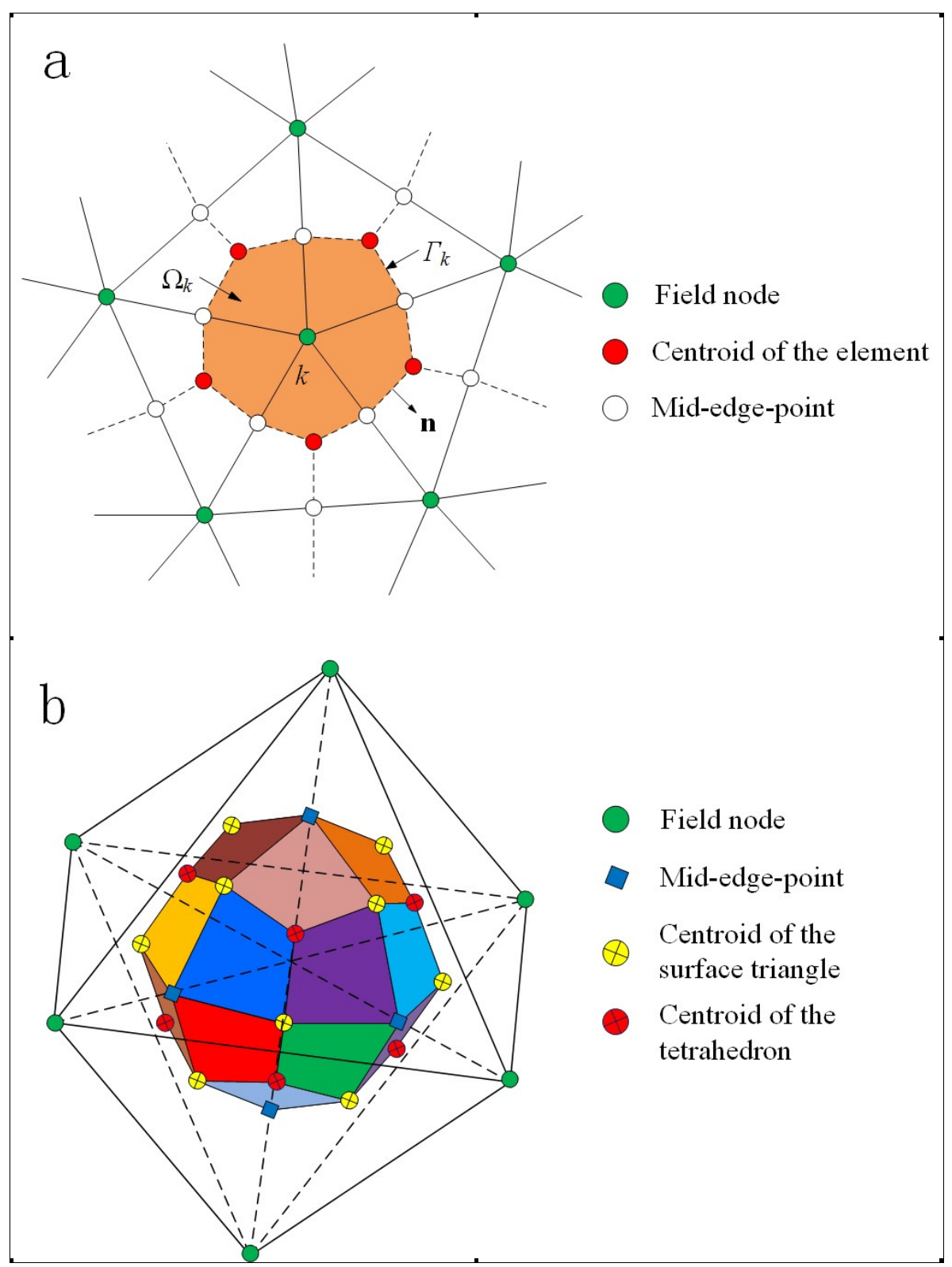

Fig.1. The schematic of a node-based smoothing domain for node $k$. (a) 2D smoothing domain, and (b) 3D smoothing domain. 



Fig.2. The integration domain and integration points for NS-FEM and SNS-FEM.
(a) NS-FEM integration for 2D, (b) SNS-FEM integration for 2D,
(c) NS-FEM integration for 3D, and (d) SNS-FEM integration for 3D. 


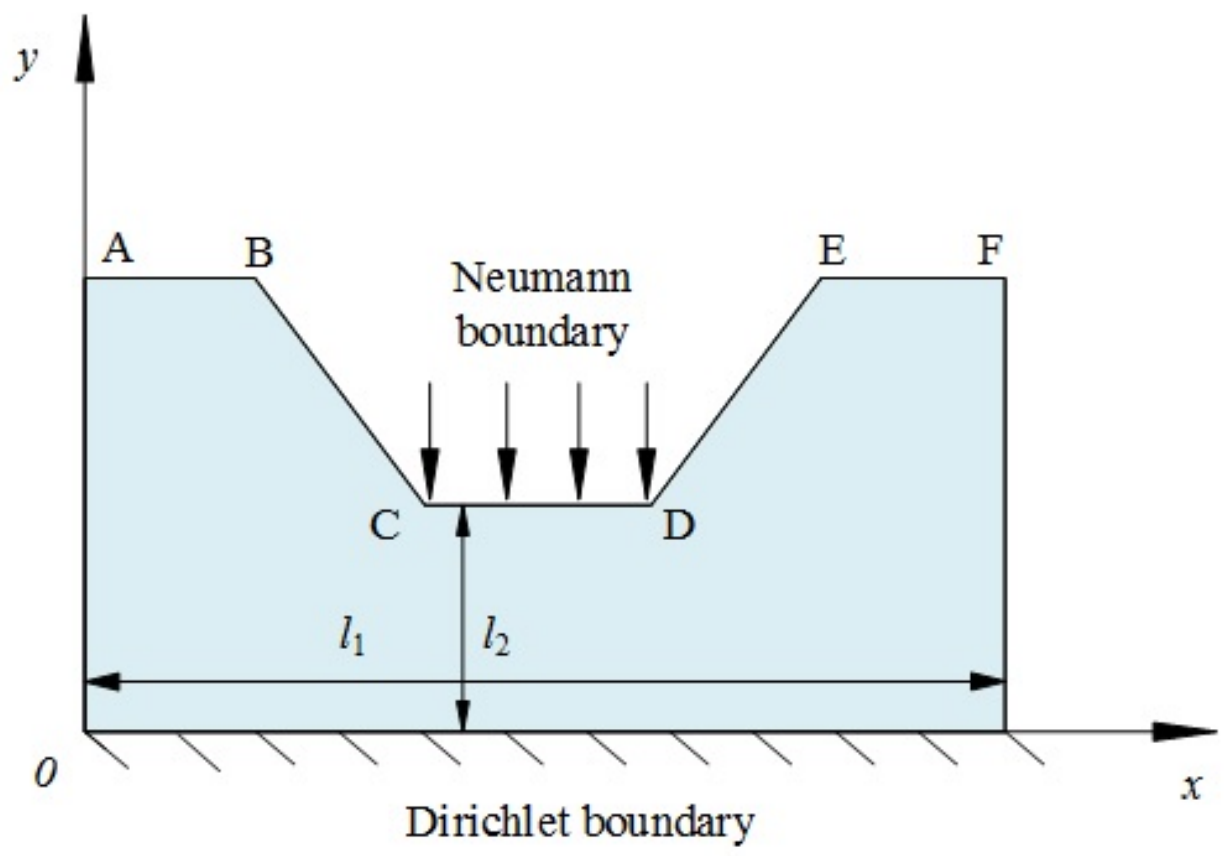

Fig.3. Schematics of a 2D steady heat transfer problem.

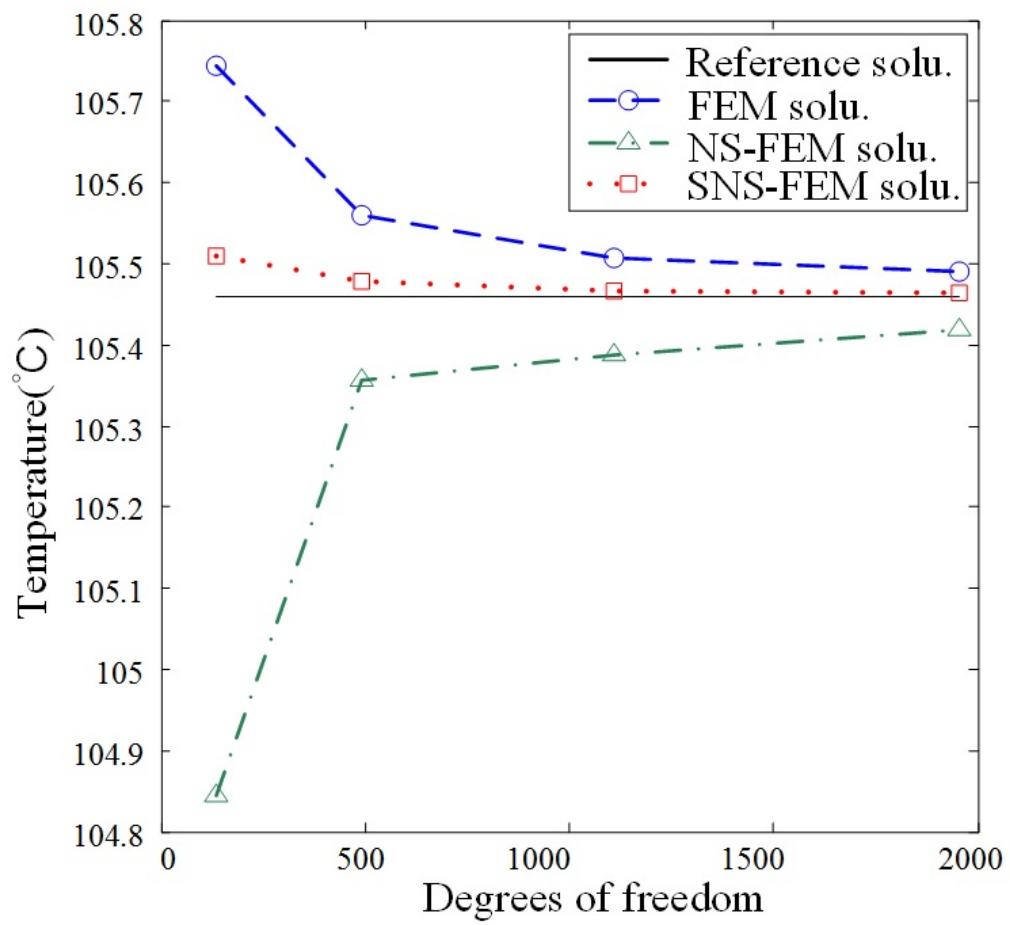

Fig. 4. Convergence of temperature versus system dof of reference point A. 


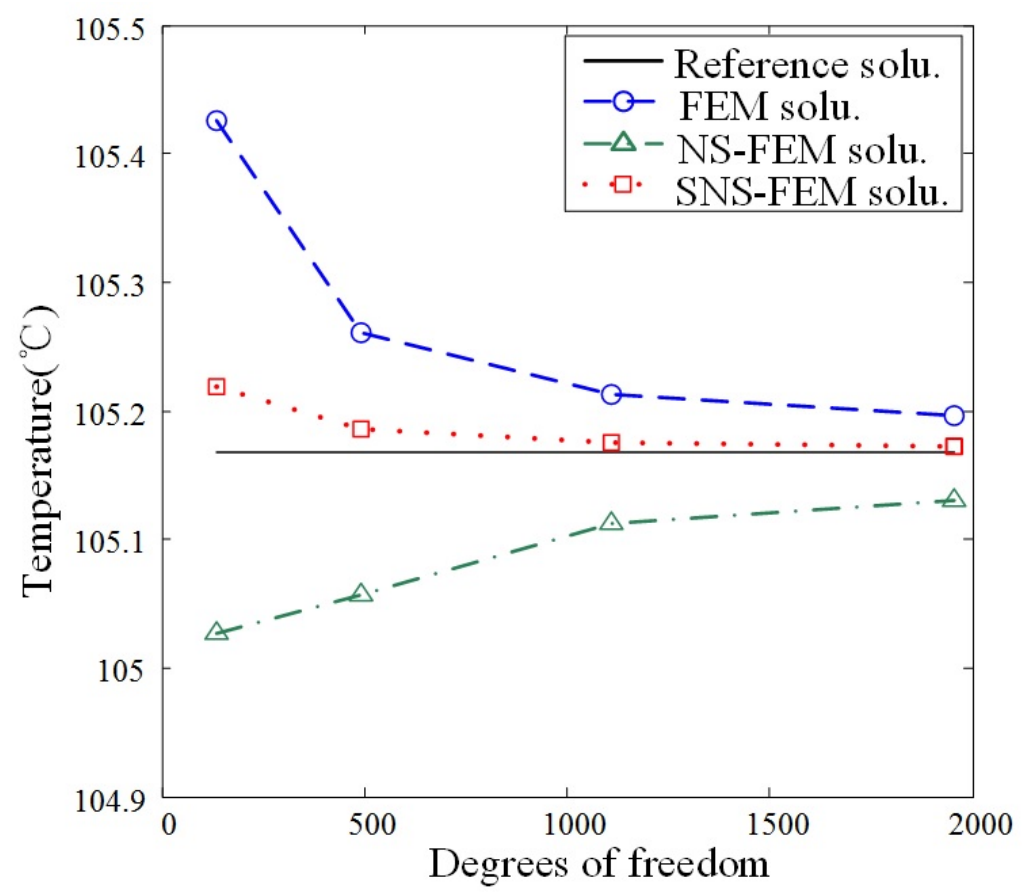

Fig.5. Convergence of temperature versus system dof of reference point B.

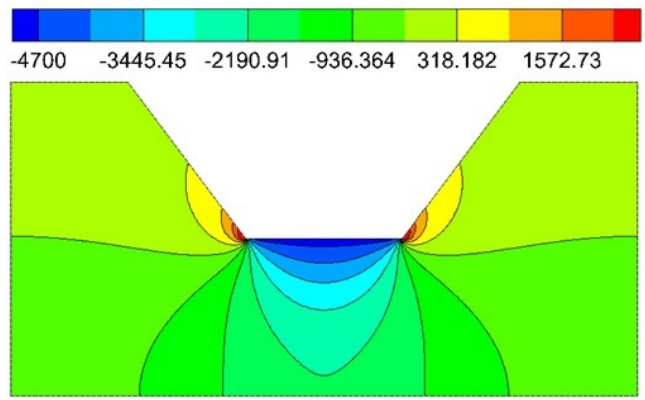

Reference solu.

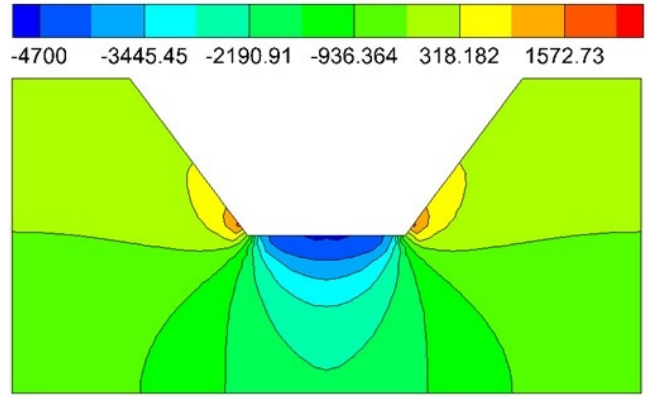

FEM solu.



SNS-FEM solu.

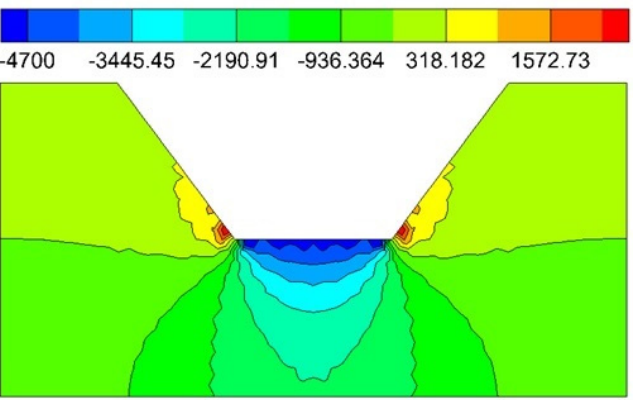

NS-FEM solu.

Fig. 6. Comparison of temperature gradient in the $\mathrm{x}$-direction $(\mathrm{l} / \mathrm{m})$. 


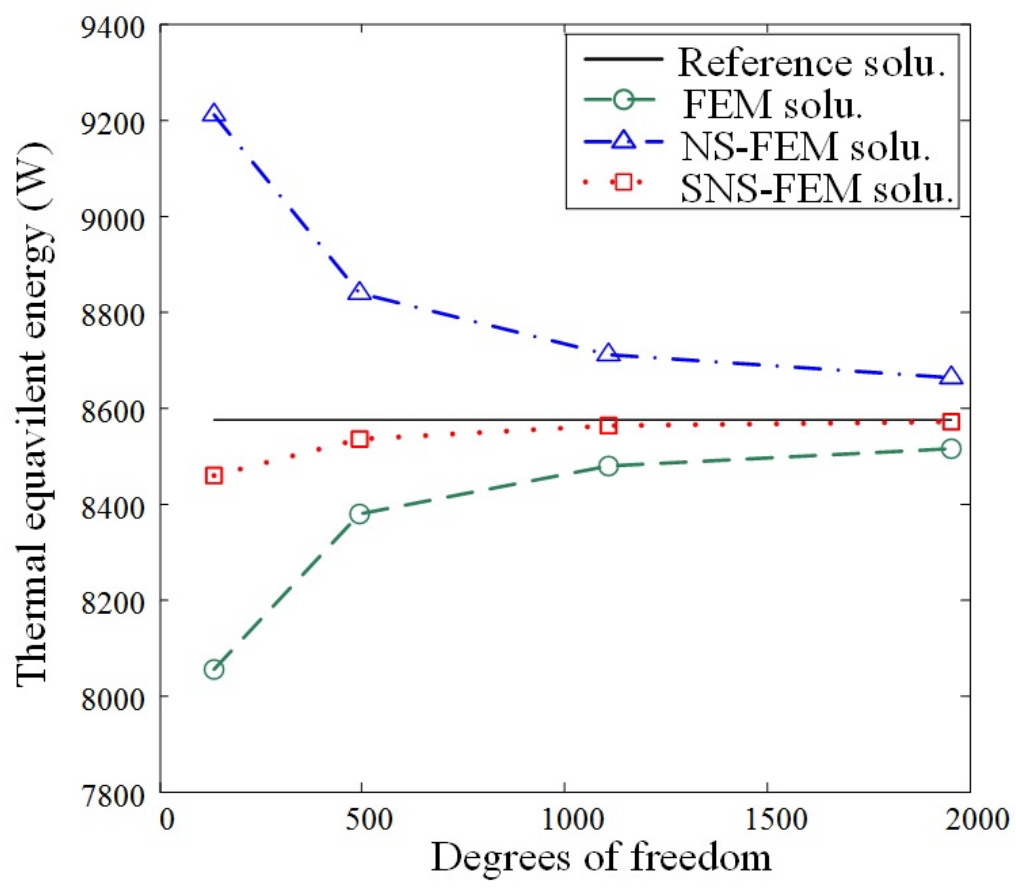

Fig. 7. Convergence of equivalent energy versus system dof. 


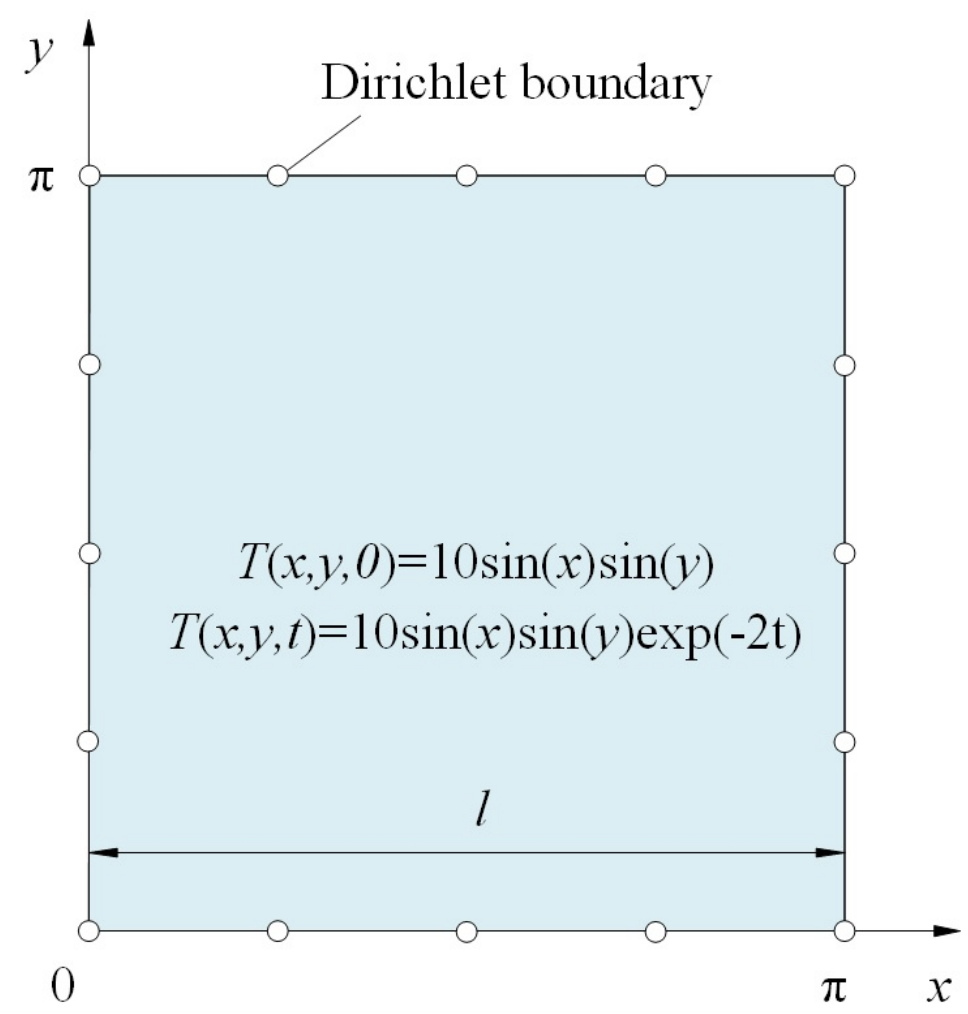

Fig. 8. Geometry and boundary conditions of the square plate.

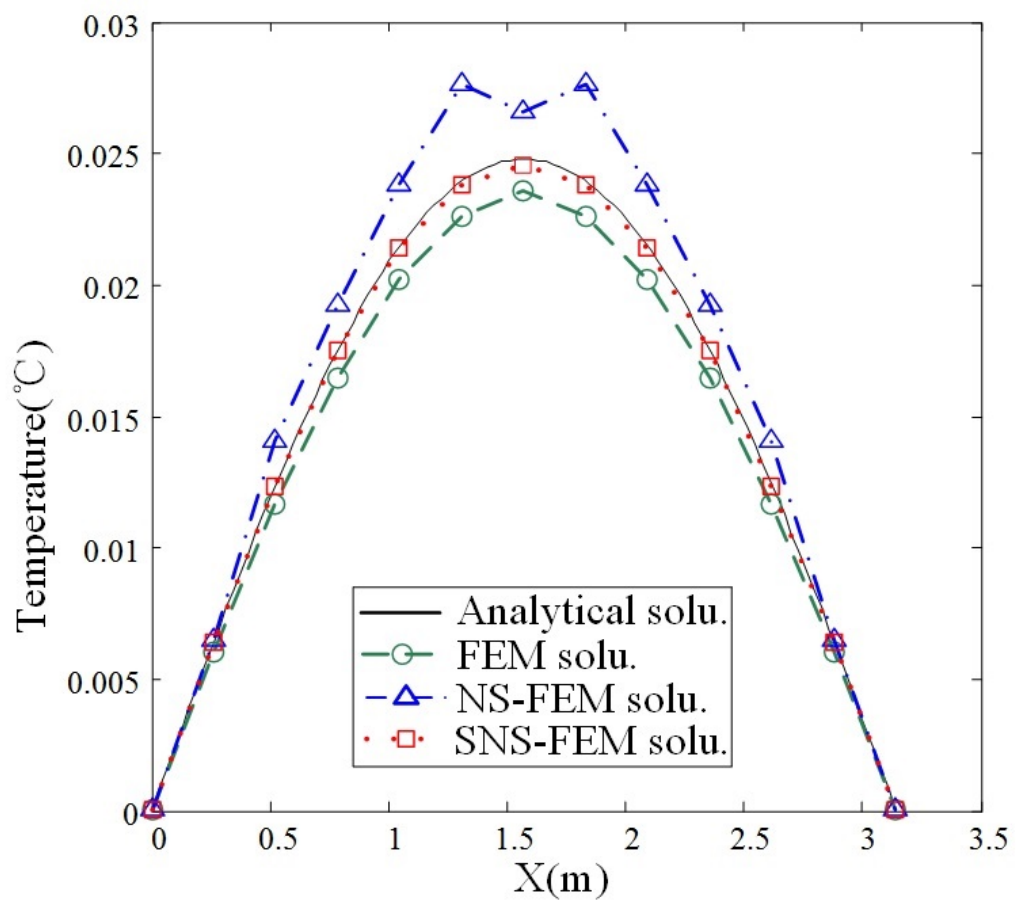

Fig. 9.Temperature distribution along $y=\pi / 2$ at $\mathrm{t}=3 \mathrm{~s}$ 


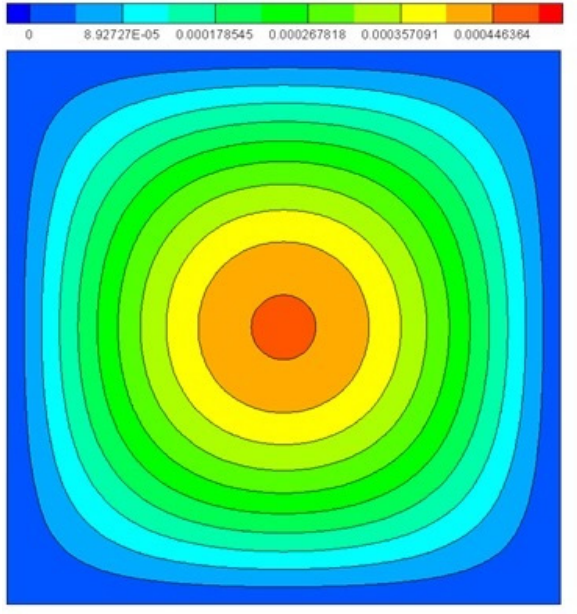

Reference solu.

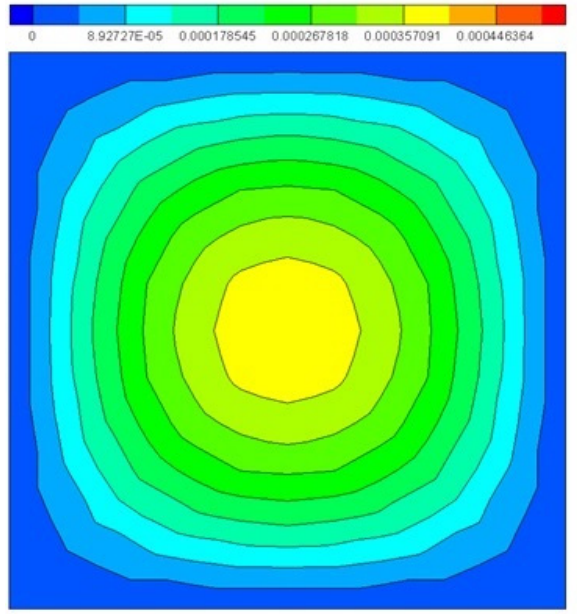

FEM solu.

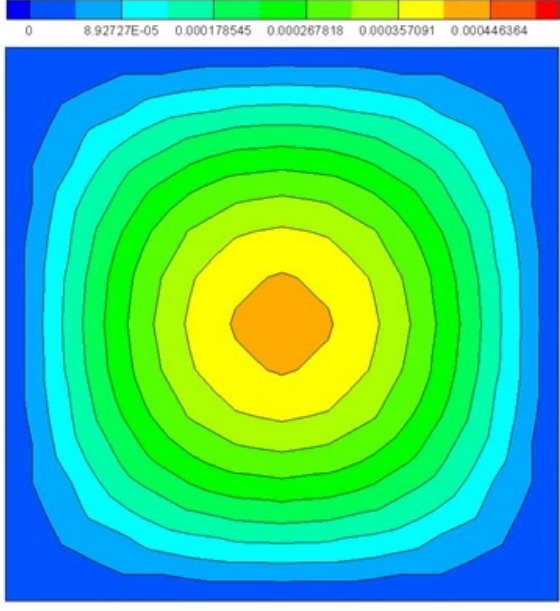

SNS-FEM solu.

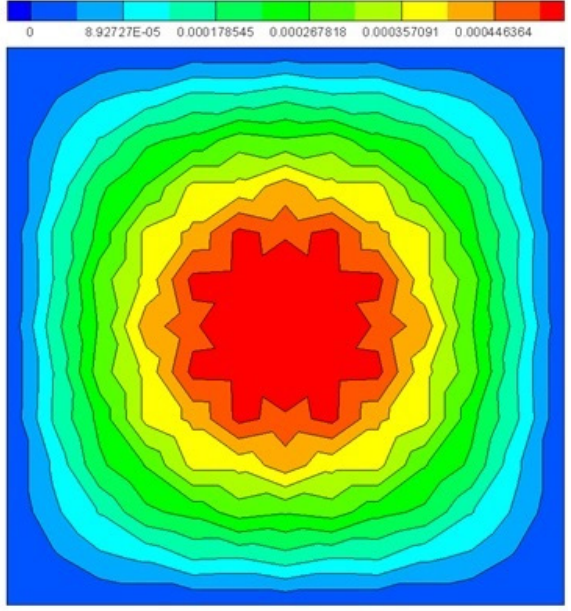

NS-FEM solu.

Fig. 10. Comparison of the temperature fields at $t=5 \mathrm{~s}$. 


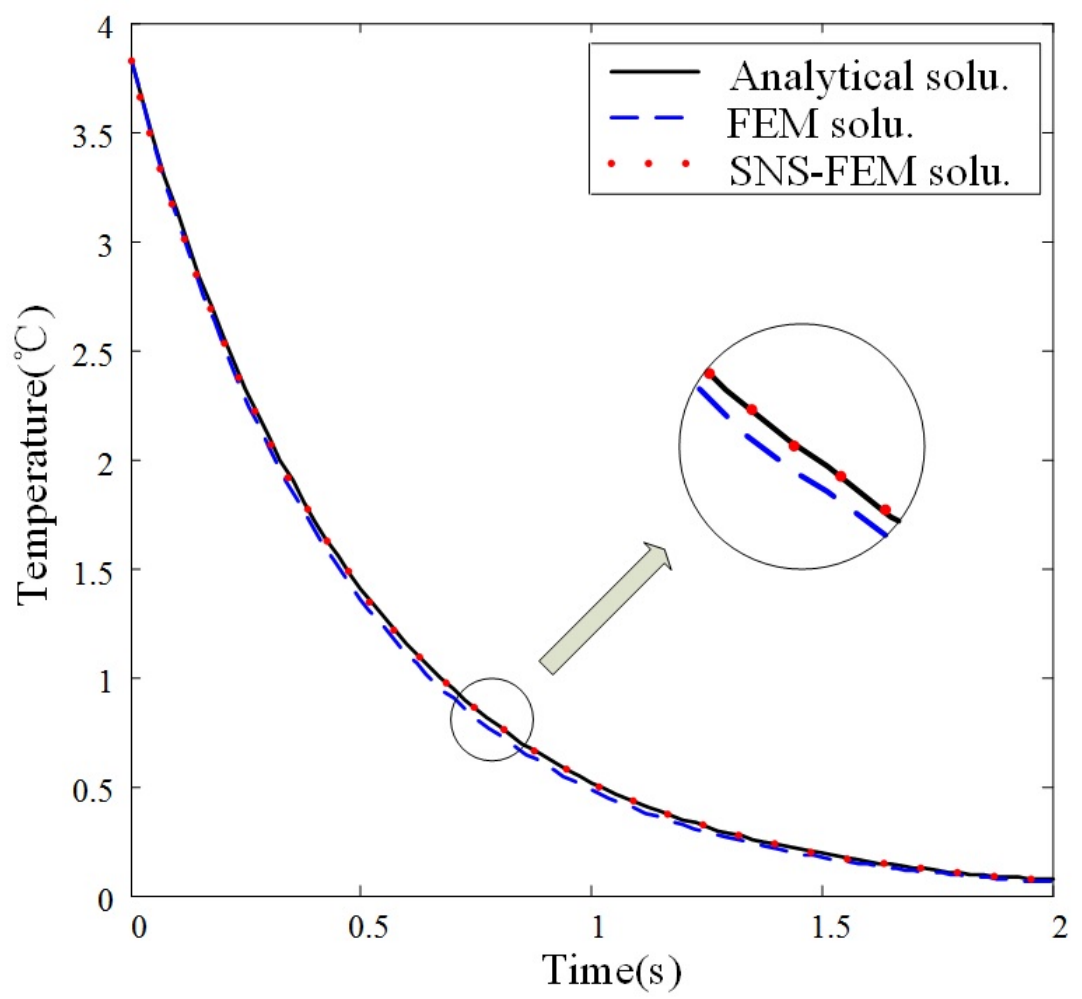

Fig. 11. Comparison of temperature history at point $(x, y)=(\pi / 2, \pi / 8)$

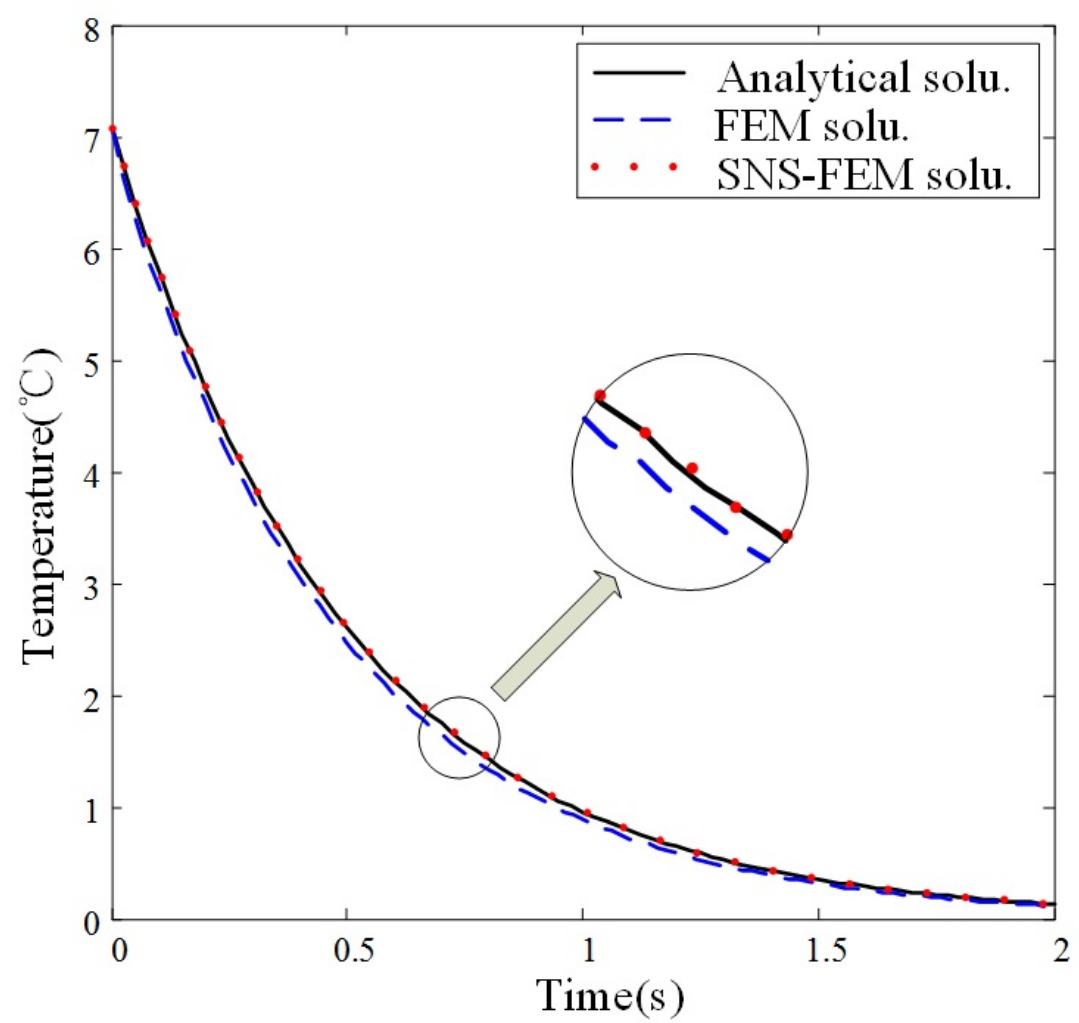

Fig. 12. Comparison of temperature history at point $(x, y)=(\pi / 2,2 \pi / 8)$ 


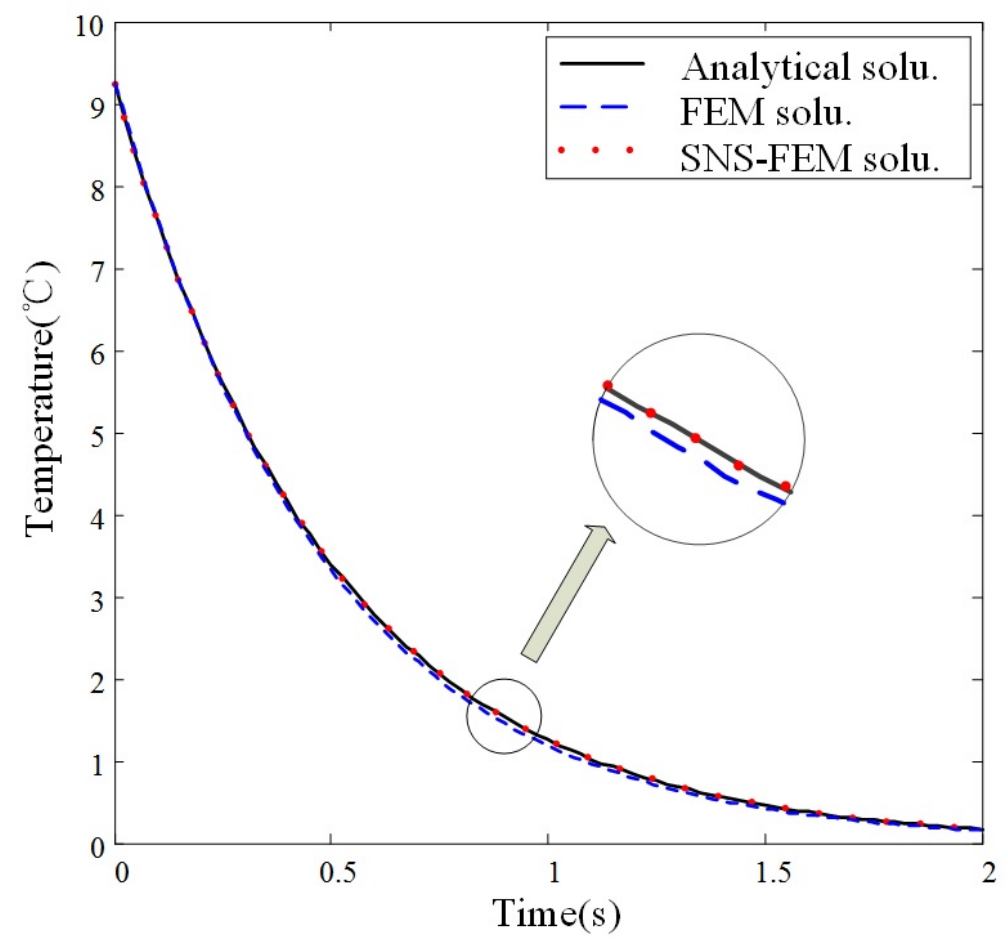

Fig. 13. Comparison of temperature history at point $(x, y)=(\pi / 2,3 \pi / 8)$

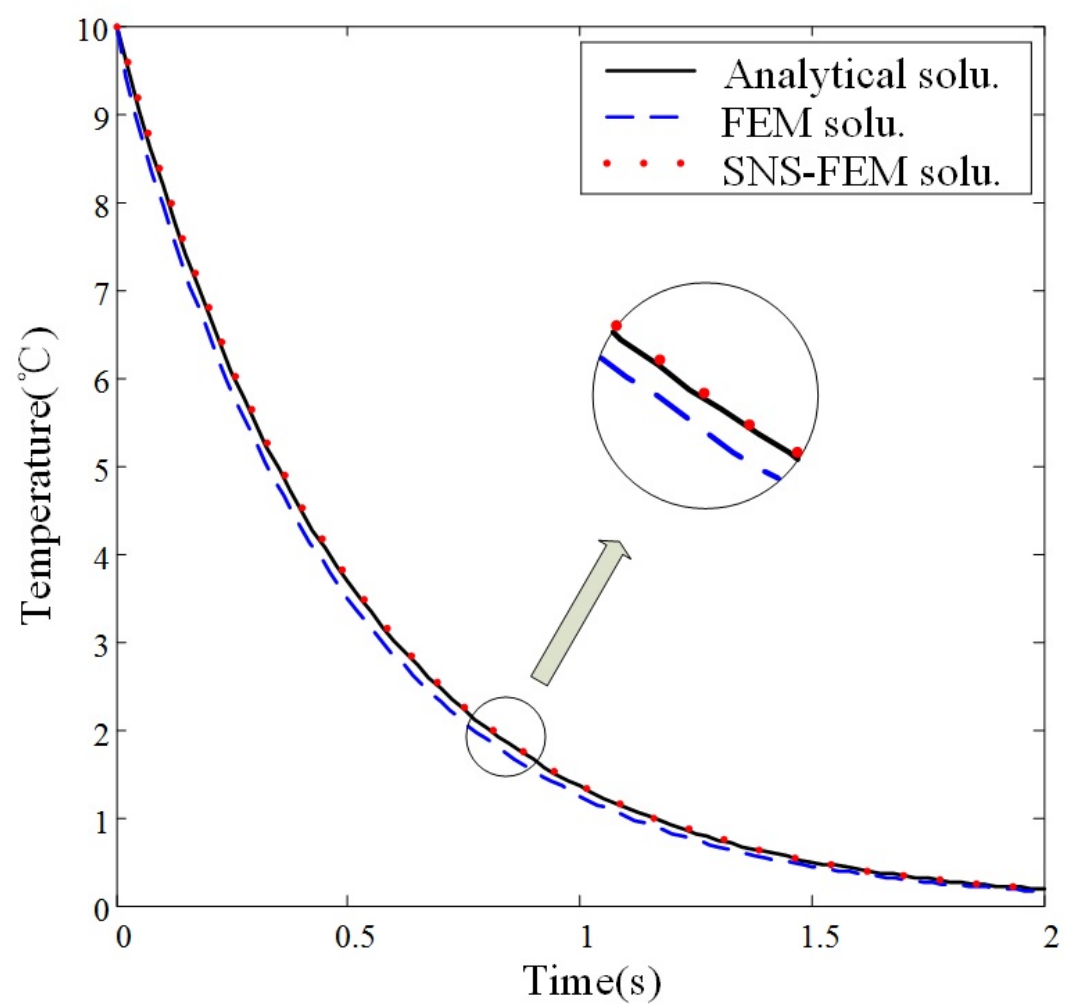

Fig. 14. Comparison of temperature history at point $(x, y)=(\pi / 2,4 \pi / 8)$ 


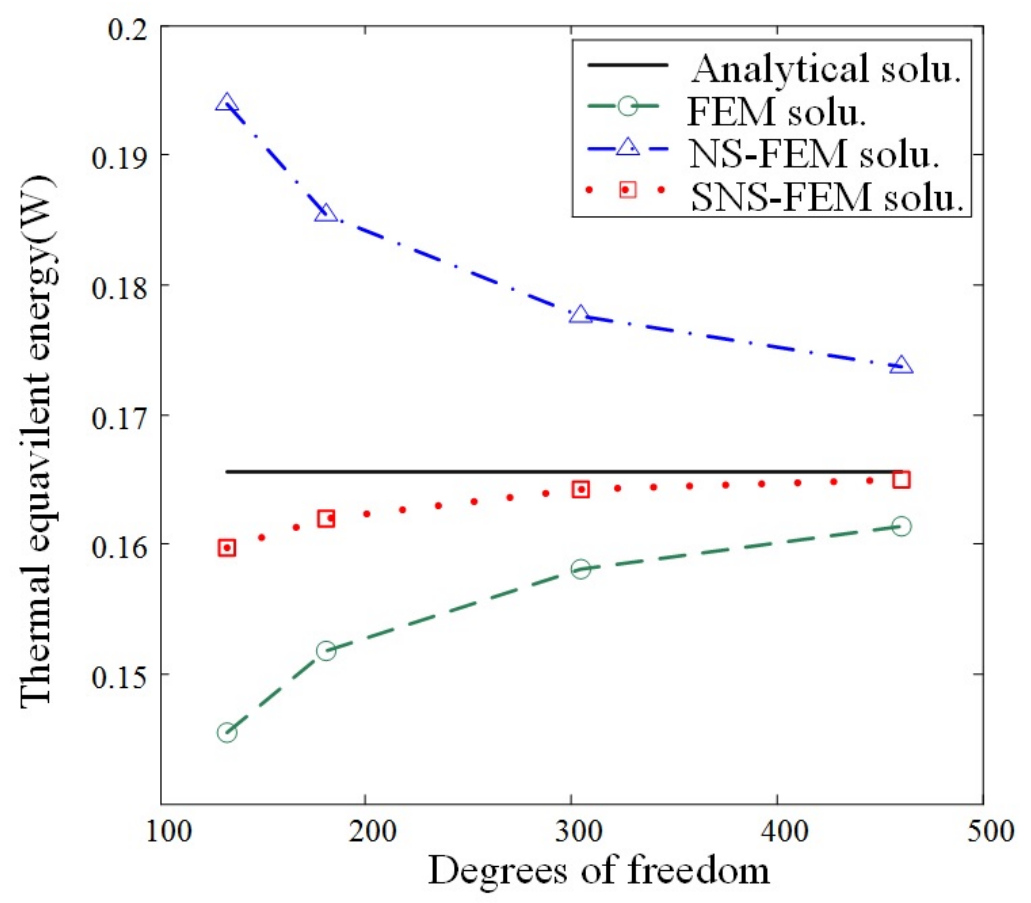

Fig. 15. Convergence of the equivalent energy versus system dof at $\mathrm{t}=2 \mathrm{~s}$.

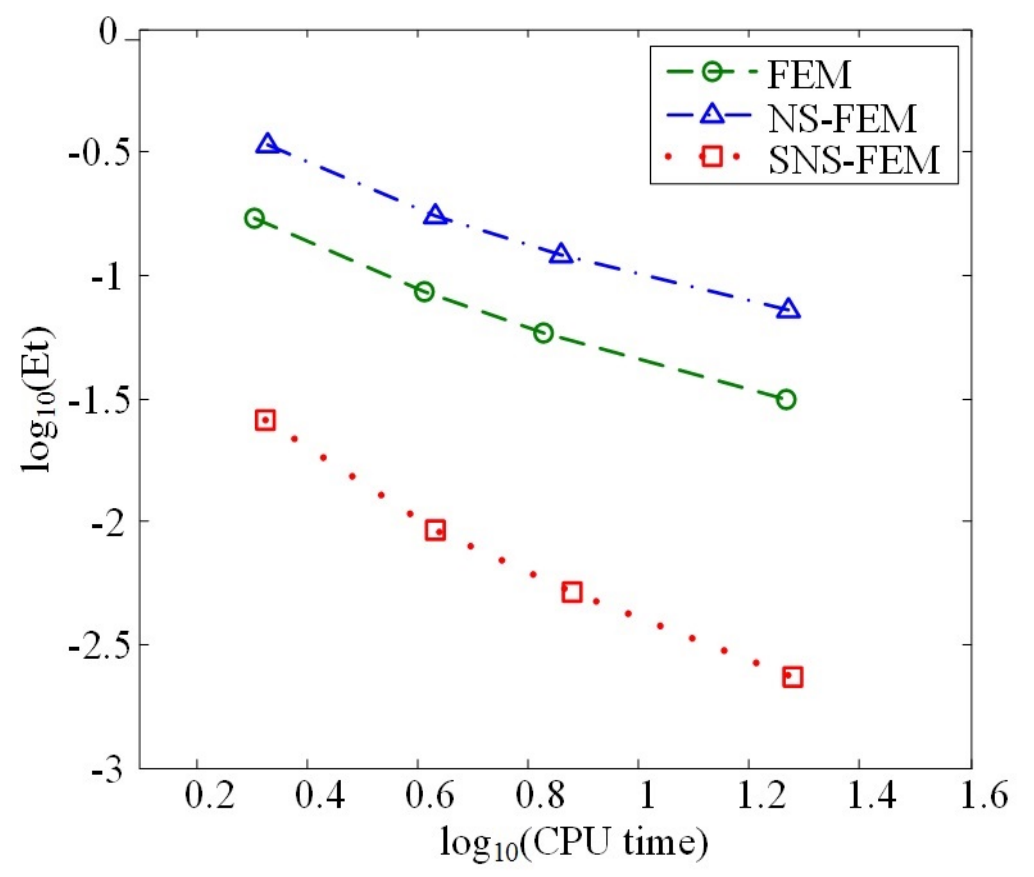

Fig. 16. Comparison of computation efficiency between FEM, NS-FEM and SNS-FEM for a range of meshes. 
Heat convective boundary

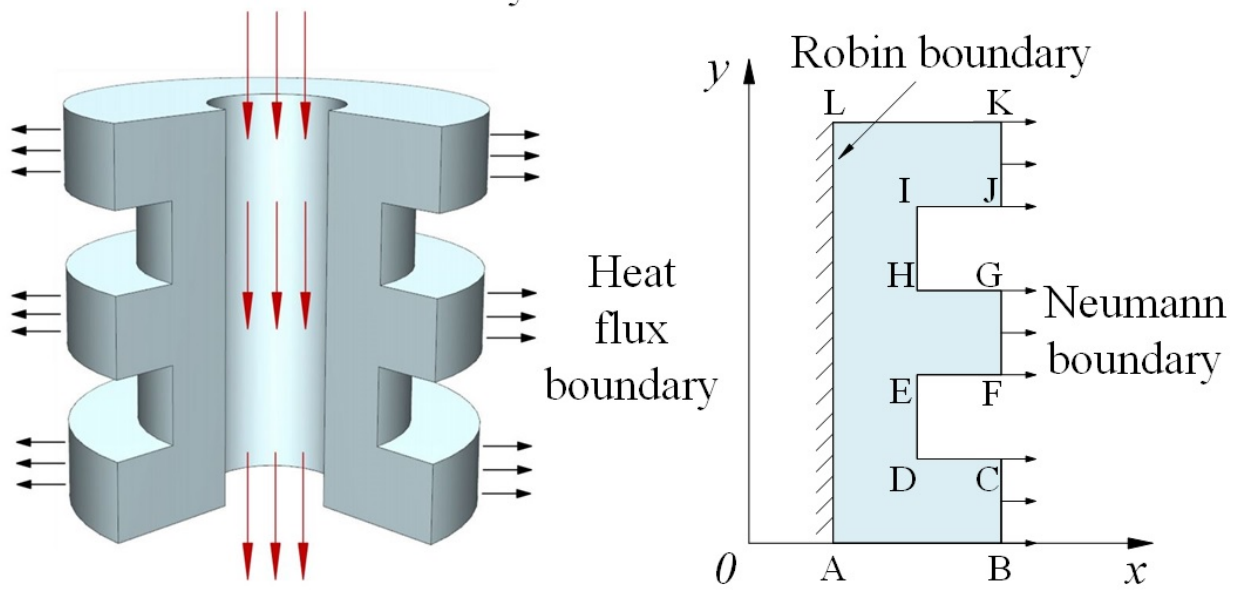

Fig. 17. Geometry and boundary conditions of the axisymmetric model. 


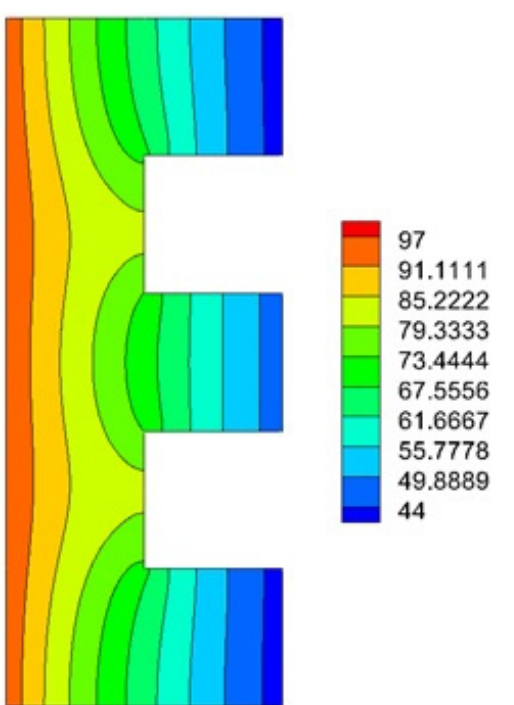

Reference solu.

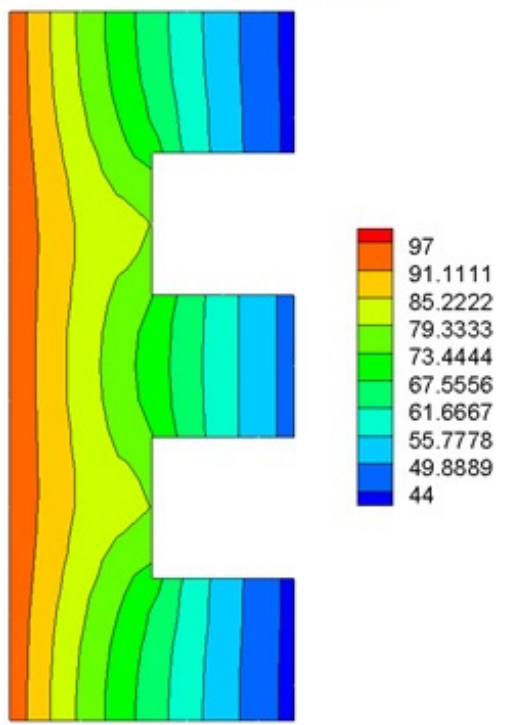

FEM solu.

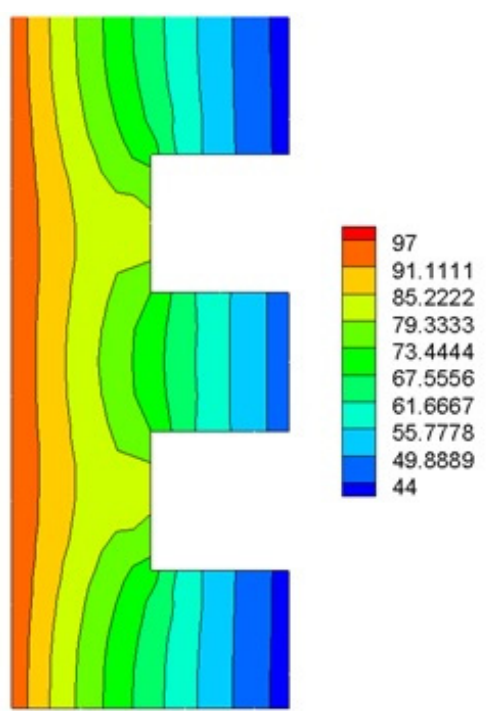

SNS-FEM solu.

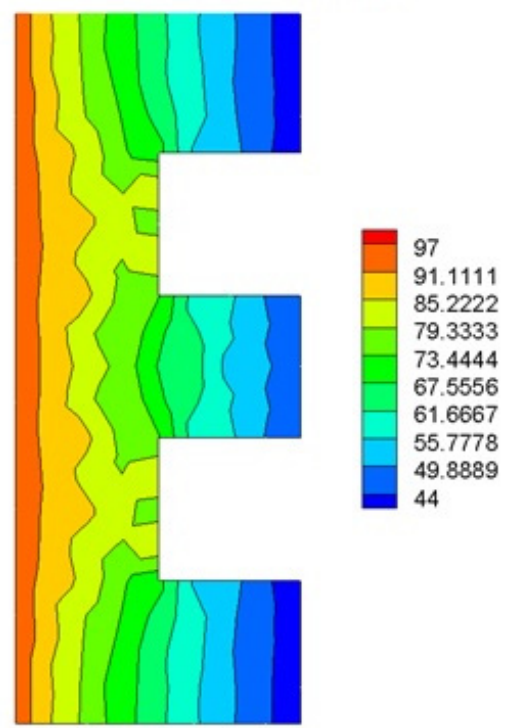

NS-FEM solu.

Fig. 18. Comparison of the temperature fields at $\mathrm{t}=5 \mathrm{~s}$. 


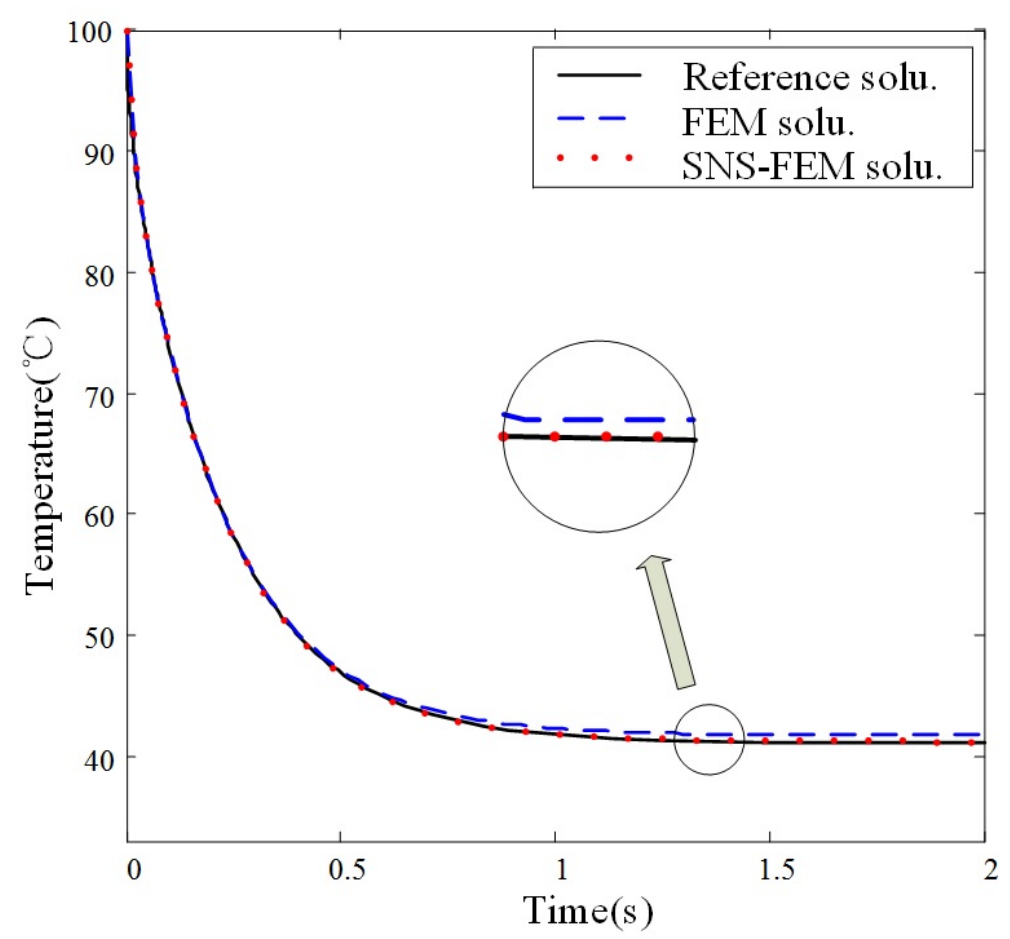

Fig. 19. Comparison of temperature history at reference point B.

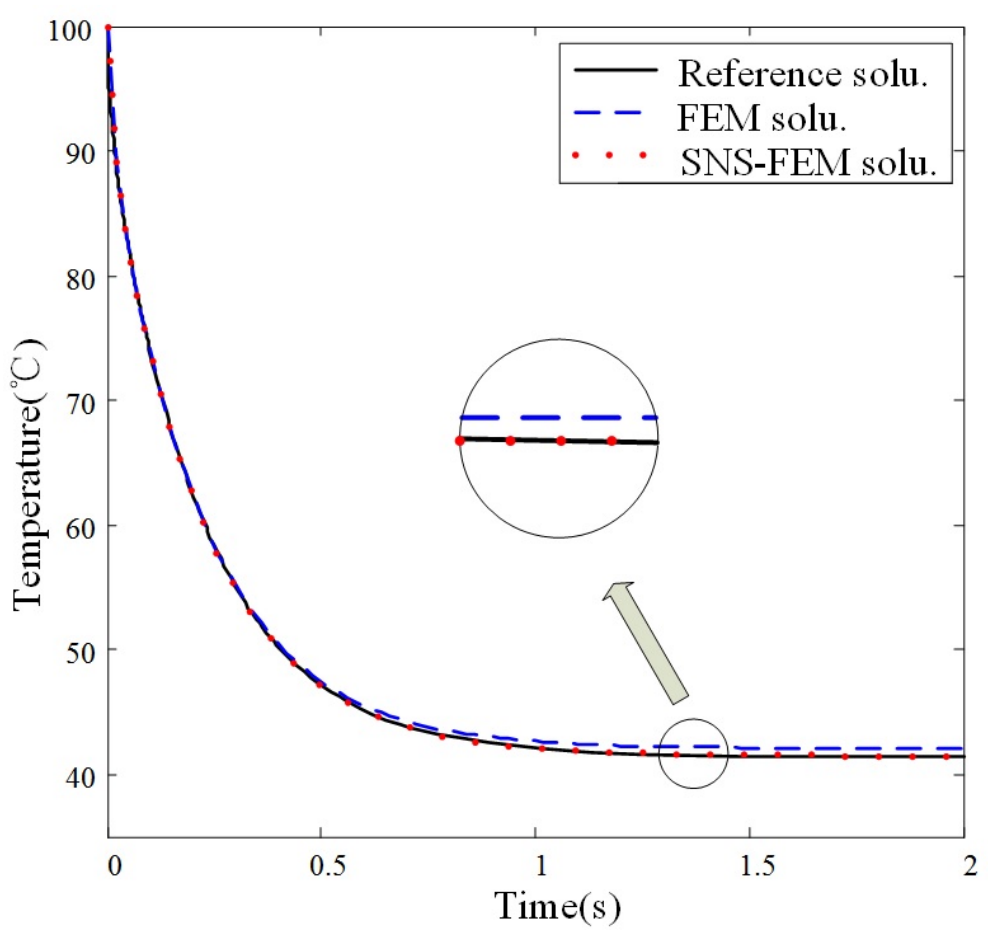

Fig. 20. Comparison of temperature history at reference point $C$.

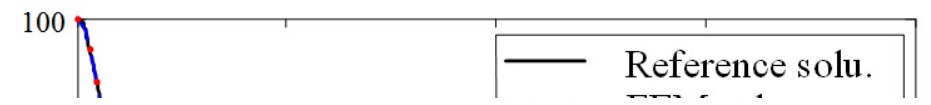


Fig. 21. Comparison of temperature history at reference point E.

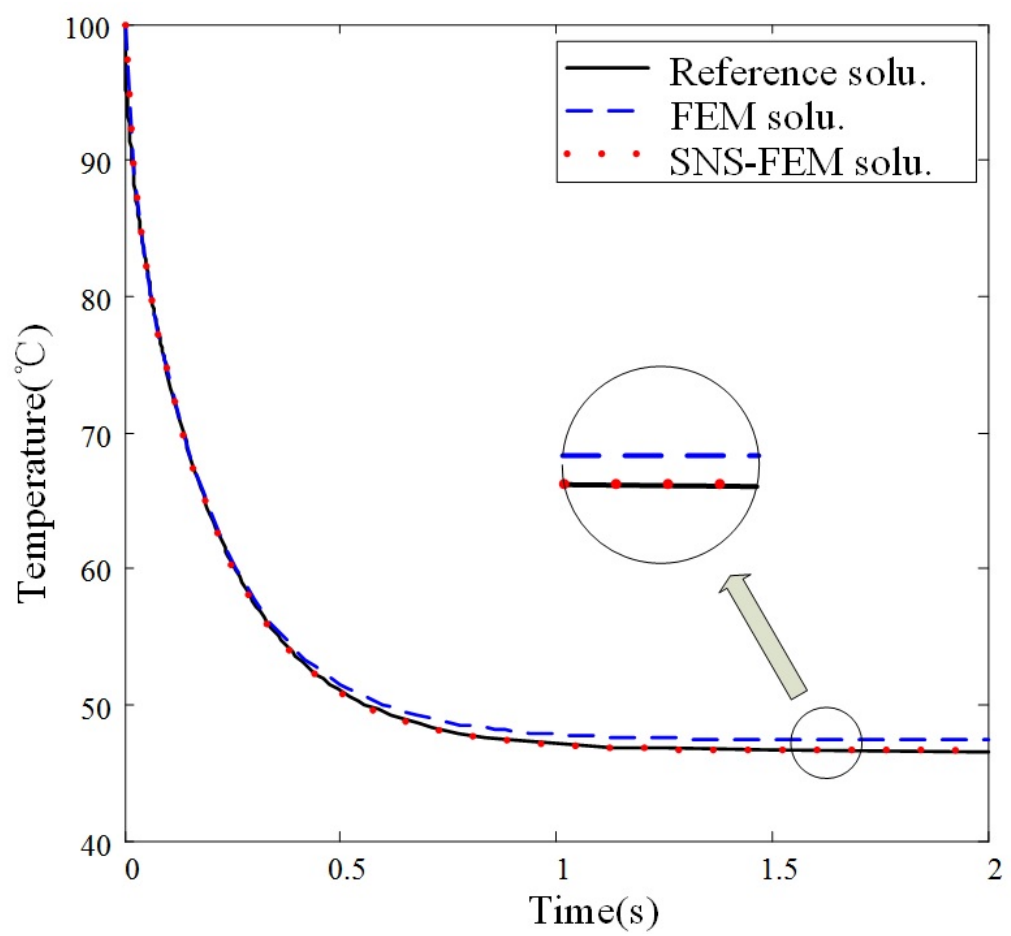

Fig. 22. Comparison of temperature history at reference point F. 


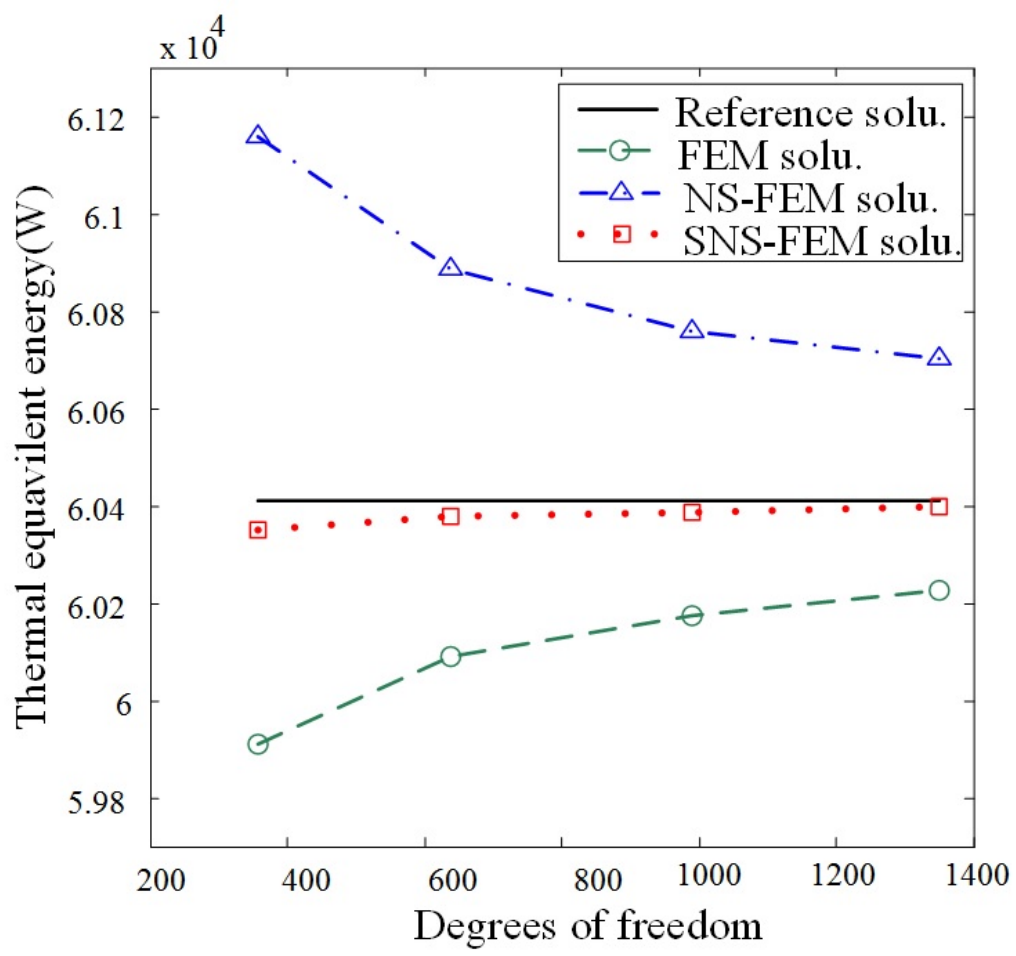

Fig. 23. Convergence of the equivalent energy versus system dof after the system stabilize. 

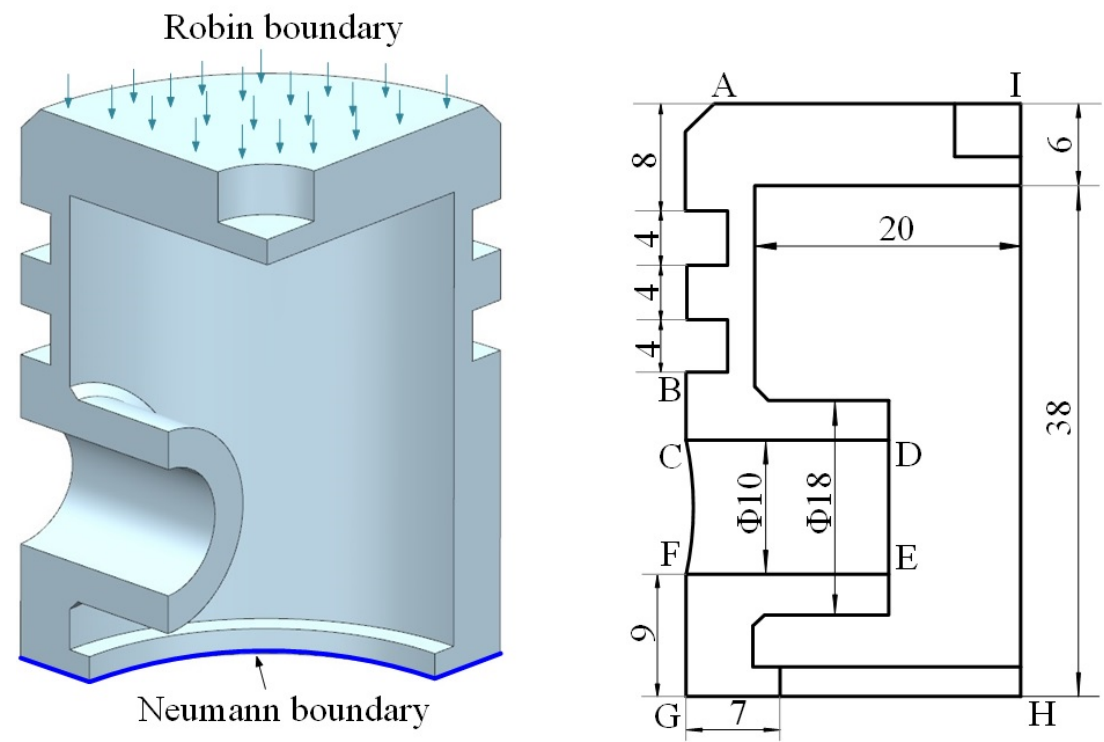

Fig. 24. Geometry and boundary conditions of a quarter of the piston model.

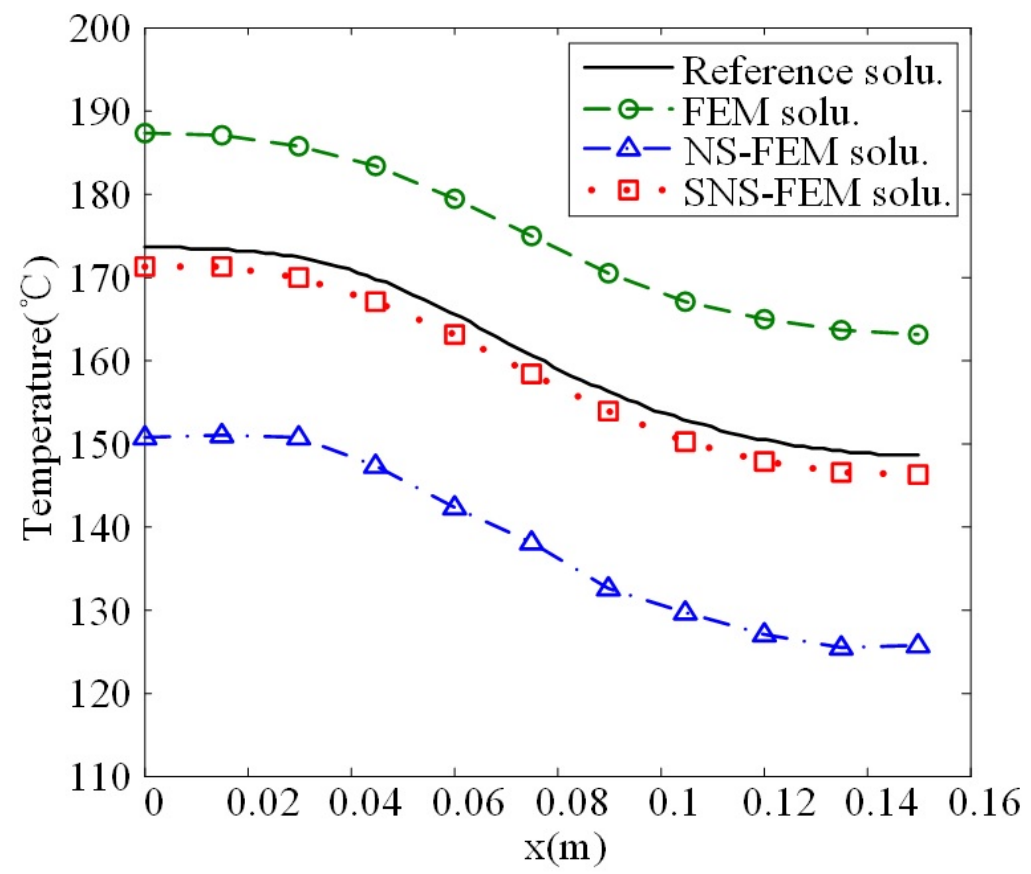

Fig. 25. Comparison of temperature distribution along CD line. 


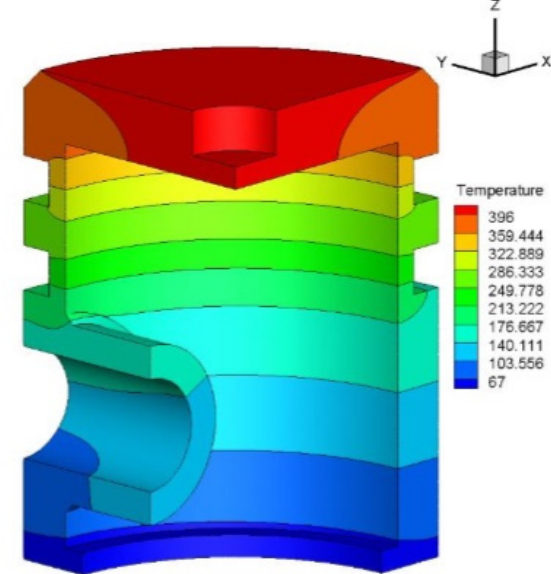

Reference solu.

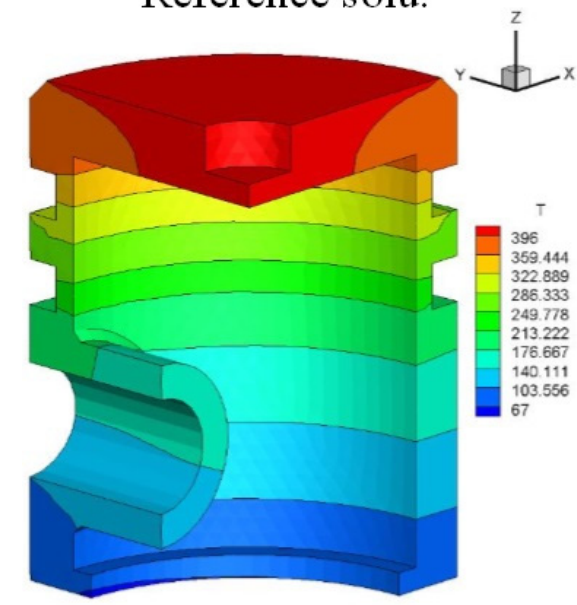

FEM solu.

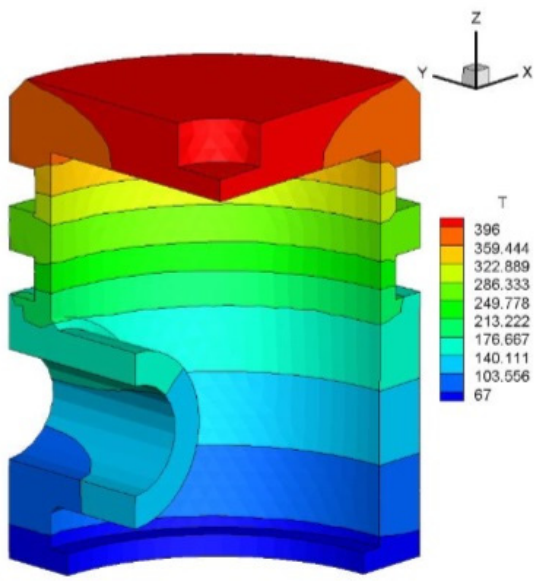

SNS-FEM solu.

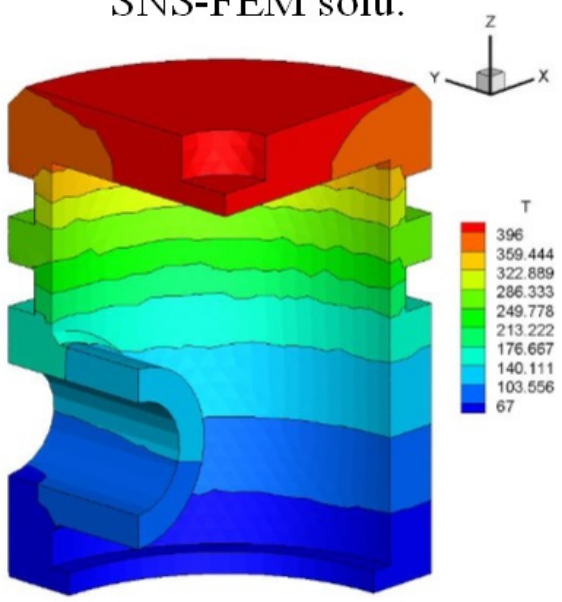

NS-FEM solu.

Fig. 26. Comparison of temperature fields. 


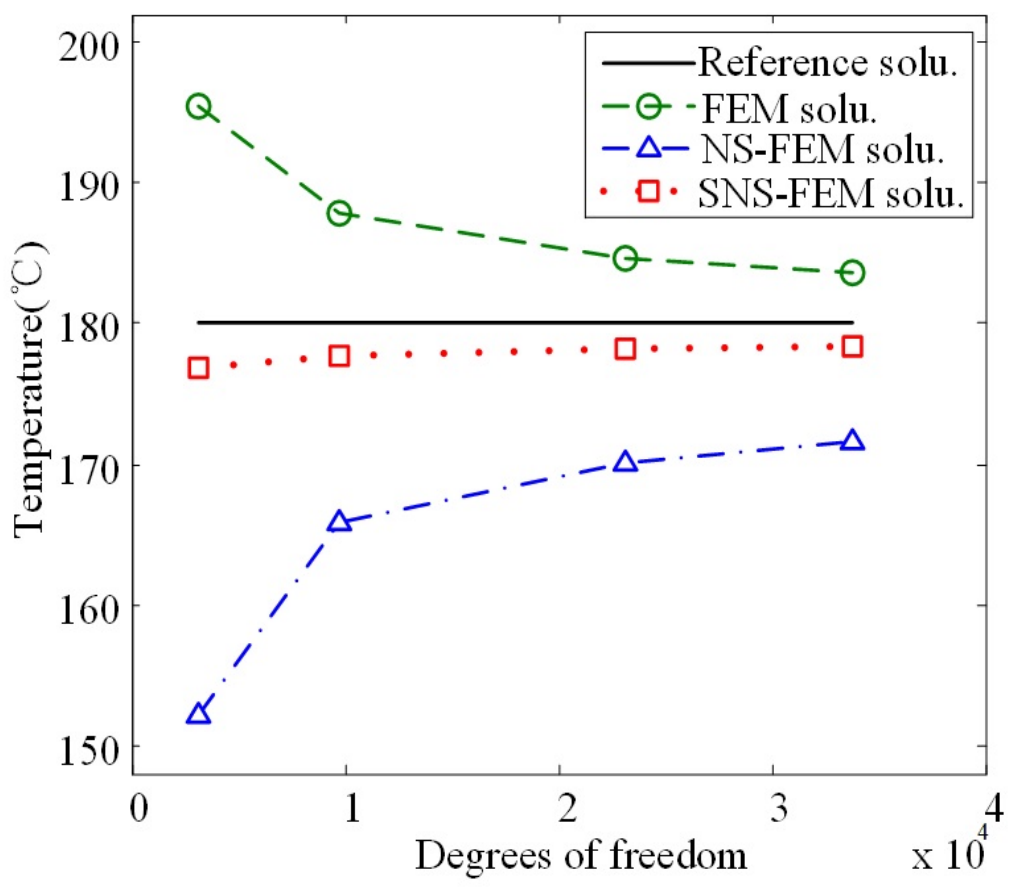

Fig. 27. Convergence of temperature versus system dof of reference point $B$.

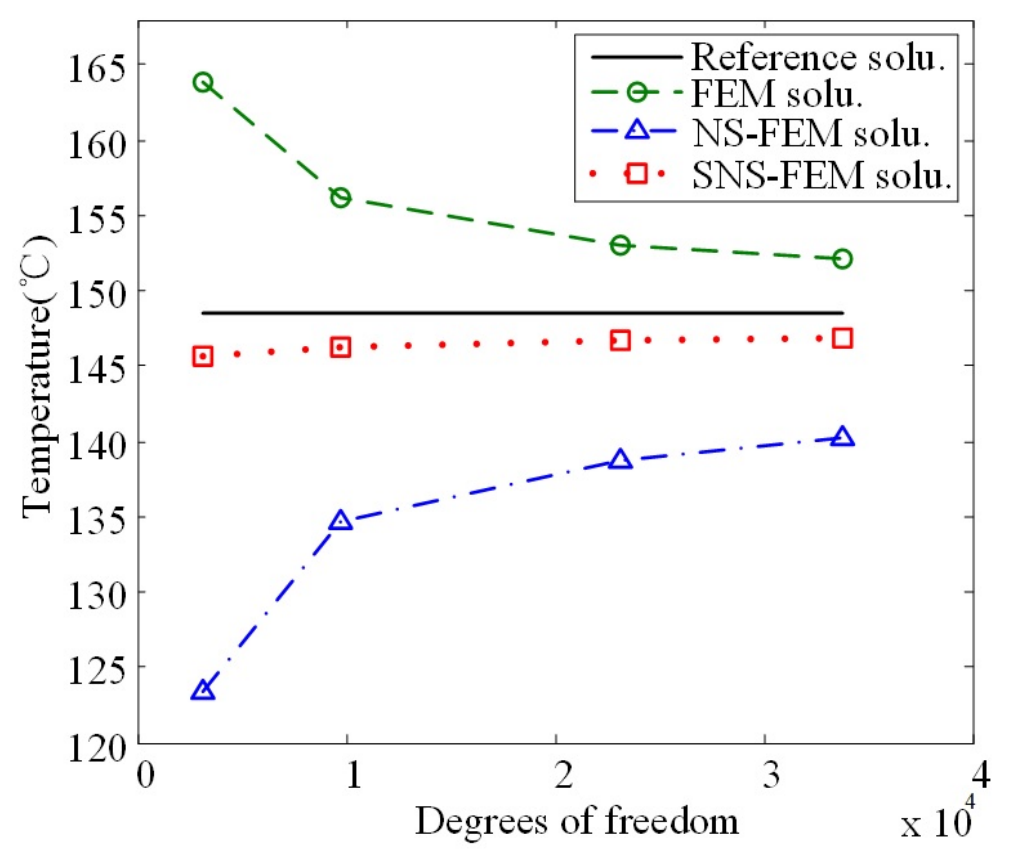

Fig. 28. Convergence of temperature versus system dof of reference point $\mathrm{F}$. 


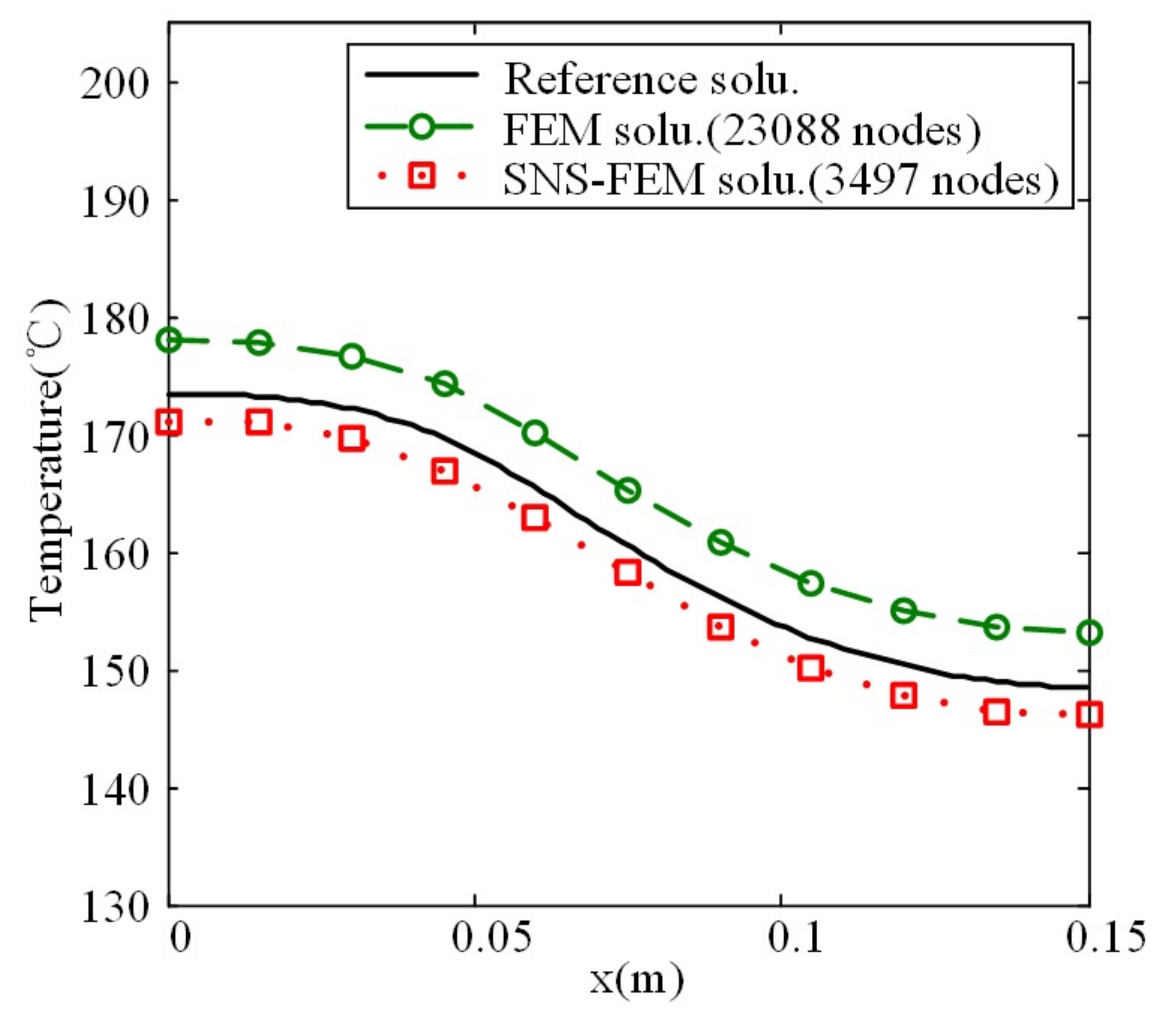

Fig. 29. Temperature distribution along CD line obtained by FEM (23088 nodes) and SNS-FEM (3497 nodes). 